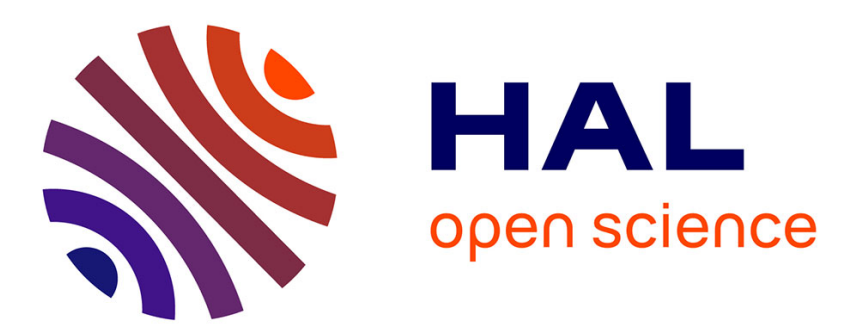

\title{
Fractional-step schemes for the coupling of distributed and lumped models in hemodynamics
}

Cristobal Bertoglio, Alfonso Caiazzo, Miguel Angel Fernández

\section{To cite this version:}

Cristobal Bertoglio, Alfonso Caiazzo, Miguel Angel Fernández. Fractional-step schemes for the coupling of distributed and lumped models in hemodynamics. SIAM Journal on Scientific Computing, 2013, 35 (3), pp.551-575. 10.1137/120874412 . hal-00690493v2

\section{HAL Id: hal-00690493 \\ https://hal.inria.fr/hal-00690493v2}

Submitted on 6 Feb 2013

HAL is a multi-disciplinary open access archive for the deposit and dissemination of scientific research documents, whether they are published or not. The documents may come from teaching and research institutions in France or abroad, or from public or private research centers.
L'archive ouverte pluridisciplinaire HAL, est destinée au dépôt et à la diffusion de documents scientifiques de niveau recherche, publiés ou non, émanant des établissements d'enseignement et de recherche français ou étrangers, des laboratoires publics ou privés. 
Fractional-step schemes for the coupling of distributed and lumped models in hemodynamics

Cristóbal Bertoglio, Alfonso Caiazzo, Miguel A. Fernández

RESEARCH

REPORT

$\mathrm{N}^{\circ} 7937$

April 2012

Project-Team REO 



\title{
Inita
}

\section{Fractional-step schemes for the coupling of distributed and lumped models in hemodynamics}

\author{
Cristóbal Bertoglid*, Alfonso Caiazzđ Miguel A. Fernández* \\ Project-Team REO \\ Research Report $\mathrm{n}^{\circ} 7937$ - April 2012 - 29 pages
}

\begin{abstract}
In three-dimensional (3D) blood flow simulations, lumped parameter models (0D) are often used to model the neglected parts of the downstream circulatory system. We analyze two 3D-0D coupling approaches in which a fractional-step projection scheme is used in the fluid. Our analysis shows that explicit approaches might yield numerical instabilities, particularly in the case of realistic geometries with multiple outlets. We introduce and analyze an implicitly 3D-0D coupled formulation with enhanced stability properties and which requires a negligible additional computational cost. Furthermore, we also address the extension of these methods to fluid-structure interaction problems. The theoretical stability results are confirmed by meaningful numerical experiments in patient specific geometries coming from medical imaging.
\end{abstract}

Key-words: Blood flows, Chorin-Temam projection method, fluid-structure interaction, lumped parameter models, 3D-0D coupling.

* Inria, Project-team REO. e-mail: cristobal.bertoglio@inria.fr, miguel.fernandez@inria.fr

$\dagger$ WIAS Berlin, Mohrenstrasse 39, 10117, Berlin, Germany. e-mail: caiazzo@wias-berlin.de

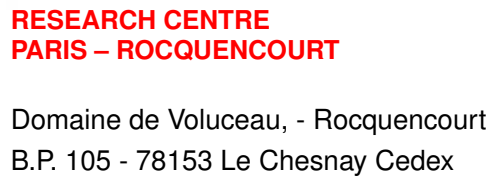




\section{Schémas à pas fractionnaire pour le couplage de modèles distribués et localisées en hémodynamique}

Résumé : Les modèles à paramètres localisés (0D) sont souvent utilisés dans les simulations tri-dimensionnelles (3D) d'écoulements sanguins pour modéliser l'effet des parties de l'appareil circulatoire négligées en aval. Dans cet article nous analysons deux approches pour le couplage de modèles 3D-0D, dans lesquelles un schéma à pas fractionnaire est utilisé dans le fluide. Notre analyse met en évidence que les schémas de couplage 3D-0D explicites peuvent donner des instabilités numériques dans le cas de sorties multiples. Nous introduisons et analysons une formulation 3D-0D implicite avec des propriétés de stabilité améliorées et un coût de calcul supplémentaire négligeable par rapport au cas explicite. Nous abordons également l'extension de ces méthodes à des problèmes d'interaction fluide-structure. Les résultats théoriques de stabilité sont confirmés par des expériences numériques dans des géométries et données réalistes.

Mots-clés : Écoulements sanguins, schéma de projection de Chorin-Temam, interaction fluidestructure, modèles à paramètres localisés, couplage 3D-0D. 


\section{Introduction}

In 3D distributed models of blood flow (e.g., Navier-Stokes equations, fluid-structure interaction), downstream pressure boundary conditions are often used to represent the effect of the neglected portion of the vessels. In fact, since pressure measurements are invasive and not always available, the downstream circulation is usually modeled trough lumped parameter (or 0D) models. This results in a set of algebraic-differential equations, relating fluxes and pressures at each outlet boundary of the fluid domain (see, e.g., [11, 21, 20, 24, and the references therein). A widely used lumped parameter model is the so-called three-element Windkessel system (see, e.g., [11, 12])

The 3D-0D coupling between distributed and lumped models is operated by interface conditions that guarantee the continuity of the fluxes and pressures on the outlet boundaries (3D-0D interfaces). It is well-known that, when this coupling is treated explicitly in time, the whole system might suffer from numerical instability, regardless of the solution scheme in the 3D compartment. This enforces restrictions on the time-step length, that can considerably increase computational cost in realistic applications (see, e.g., the discussion in [21]). On the other hand, implicit 3D-0D coupling schemes overcome this instability issue at the expense of solving a fully coupled 3D-0D system at each time step. The so-called partitioned approaches solve this system by sub-iterating between the 3D and 0D models, which might be inefficient in practice (see, e.g., [18]). Alternatively, monolithic procedures solve both models simultaneously and yield system matrices with a modified sparsity pattern, which might lead to preconditioning issues (see, e.g., [19]).

This work is devoted to the formulation and analysis of 3D-0D coupling schemes based on a fractional-step projection time-marching of the fluid (see, e.g., 6, 15, 23]). First, an advectiondiffusion problem is solved to recover an intermediate approximation of the velocity field and, then, a suitable pressure field is recovered by solving a Poisson problem. We also consider the case of a 3D fluid-structure interaction modeling, time-discretized by the projection semiimplicit coupling scheme reported in 9. In this fractional-step framework, the explicit and implicit treatments of the 3D-0D coupling lead to different formulations of the pressure-Poisson projection step. It can be discretized in an explicit fashion by time-advancing the 0D model from the previously computed intermediate velocity flux, which provides an explicit Dirichlet boundary data for the 3D pressure-Poisson problem. For a purely fluid 3D distributed modeling with two or more 3D-0D interfaces, our analysis shows that this approach might compromise stability. We also show that, in the case of fluid-structure interaction, numerical instabilities might appear even with a sole downstream boundary. We propose to overcome these instability issues through the introduction of a implicit 3D-0D formulation with enhanced energy balance across the 3D0D interface, both for a fluid and a fluid-structure interaction modeling of blood flow. A salient feature of the proposed schemes is that they preserve the two-step velocity/pressure splitting of the original fractional-step scheme, characterized by a 3D-0D coupled problem of reduced size. Moreover, we show that this coupling strategy can be efficiently implemented by considering a single unknown per 3D-0D interface. This yields a computational complexity comparable to an explicit scheme and, hence, overcomes the above mentioned stability/complexity issues.

The rest of the paper is organized as follows. In Section 2 we introduce the 3D fluid equations, its corresponding time discretization (via a fractional-step projection scheme) and the considered Windkessel model. Section 3 is devoted to the formulation and analysis of explicit and implicit 3D-0D coupling schemes with a 3D distributed model based on the Navier-Stokes equations, while Section 4 consider the fluid-structure interaction case. In section 5 we present and discuss the performed numerical experiments, that is, the Navier-Stokes flow in a realistic aortic geometry and with measured clinical data, and the fluid-structure interaction in an ideal abdominal aortic 
aneurism. At last, Section 6 draws some concluding remarks and lines of future work.

\section{The 3D-0D model problem}

We first summarize the main ingredients of a 3D-0D model of blood flow coupling the incompressible Navier-Stokes equation and a lumped parameter description of the downstream boundaries. We then present the time-marching schemes considered in each sub-system and introduce some notation for the spatial discretization of the fluid equations.

\subsection{D fluid equations}

We consider a domain $\Omega_{\mathrm{f}} \subset \mathbb{R}^{3}$ with the following partition of its boundary $\partial \Omega_{\mathrm{f}} \stackrel{\text { def }}{=} \Gamma^{\mathrm{in}} \cup \Sigma \cup$ $\Gamma^{\text {out }}$. In the context of blood flow simulations, $\Omega_{\mathrm{f}}$ will represent the lumen of the vessel (see Figure 1), with $\Gamma^{\text {in }}, \Sigma$ and $\Gamma^{\text {out }}$ denoting, respectively, the inlet, vessel wall and outlet boundaries. We

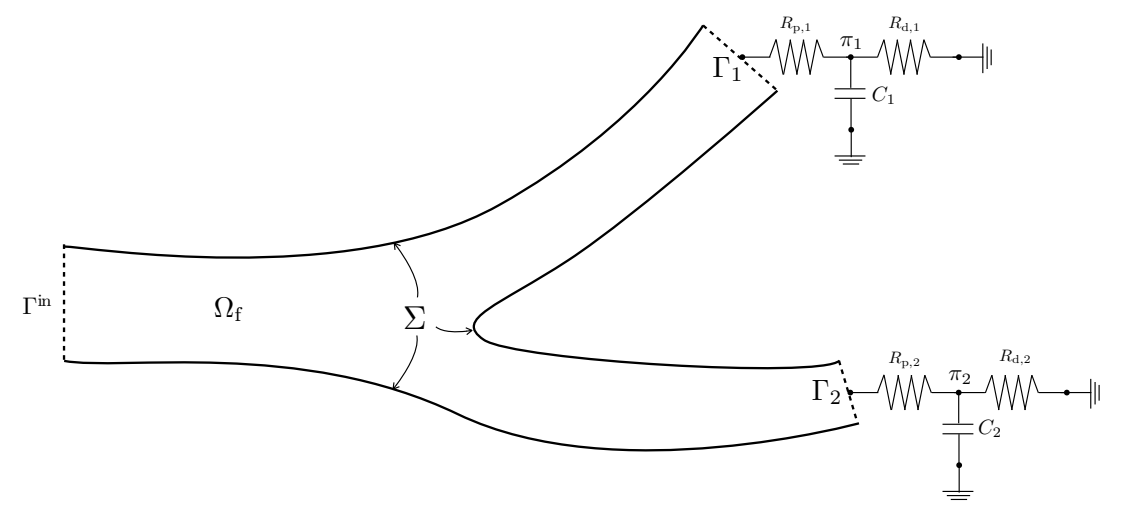

Figure 1: Sketch of the fluid domain $\Omega_{\mathrm{f}}$ with two outlet boundaries $\Gamma^{\text {out }}=\Gamma_{1} \cup \Gamma_{2},\left(n_{0 \mathrm{D}}=2\right)$.

now consider the incompressible Navier-Stokes equations for the velocity $\boldsymbol{u}: \Omega_{\mathrm{f}} \times \mathbb{R}^{+} \rightarrow \mathbb{R}^{3}$ and the pressure $p: \Omega_{\mathrm{f}} \times \mathbb{R}^{+} \rightarrow \mathbb{R}:$

$$
\left\{\begin{aligned}
\rho_{\mathrm{f}} \frac{\partial \boldsymbol{u}}{\partial t}+\rho_{\mathrm{f}} \boldsymbol{u} \cdot \boldsymbol{\nabla} \boldsymbol{u}-\boldsymbol{\nabla} \cdot \boldsymbol{\sigma}(\boldsymbol{u}, p)=\mathbf{0} & \text { in } \quad \Omega_{\mathrm{f}}, \\
\boldsymbol{\nabla} \cdot \boldsymbol{u}=0 & \text { in } \quad \Omega_{\mathrm{f}}, \\
\boldsymbol{u}=\boldsymbol{u}_{\text {in }} & \text { on } \quad \Gamma^{\text {in }}, \\
\boldsymbol{u}=\mathbf{0} & \text { on } \quad \Sigma,
\end{aligned}\right.
$$

where $\rho_{\mathrm{f}}$ stands for the density of the fluid and the fluid Cauchy-stress tensor is given by $\boldsymbol{\sigma}(\boldsymbol{u}, p) \stackrel{\text { def }}{=}-p \boldsymbol{I}+2 \mu \boldsymbol{\epsilon}(\boldsymbol{u})$ and $\boldsymbol{\epsilon}(\boldsymbol{u}) \stackrel{\text { def }}{=} \frac{1}{2}\left(\boldsymbol{\nabla} \boldsymbol{u}+\boldsymbol{\nabla} \boldsymbol{u}^{\mathrm{T}}\right), \mu$ being the dynamic viscosity of the fluid and $\boldsymbol{u}_{\text {in }}$ being a given inlet velocity field. The additional boundary conditions on $\Gamma^{\text {out }}$ will be considered in the next subsection. 


\subsection{D Windkessel model}

In what follows, the oulet boundary $\Gamma^{\text {out }}$ is assumed to be made of $n_{0 \mathrm{D}}$ components

$$
\Gamma^{\mathrm{out}}=\bigcup_{l=1}^{n_{\mathrm{OD}}} \Gamma_{l}
$$

such that $\bar{\Gamma}_{i} \cap \bar{\Gamma}_{j}=\emptyset$ for $i, j=1, \ldots, n_{0 \mathrm{D}}$ with $i \neq j$ (see, e.g., Figure 1 ). We will consider a threeelement Windkessel model (see, e.g., [11, Chapter 10] and [12]), where the pressure $P_{l}: \mathbb{R}^{+} \rightarrow \mathbb{R}$ and the flux $Q_{l}: \mathbb{R}^{+} \rightarrow \mathbb{R}$ on the outlet $\Gamma_{l}$ are related through the following algebraic-differential equations:

$$
\left\{\begin{array}{l}
C_{\mathrm{d}, l} \frac{\mathrm{d} \pi_{l}}{\mathrm{~d} t}+\frac{\pi_{l}}{R_{\mathrm{d}, l}}=Q_{l} \\
P_{l}=R_{\mathrm{p}, l} Q_{l}+\pi_{l}
\end{array}\right.
$$

for $l=1, \ldots, n_{0 \mathrm{D}}$. Here, $R_{\mathrm{p}, l}$ and $R_{\mathrm{d}, l}$ model the resistance to the flow of the vasculature proximal and distal to each outlet $\Gamma_{l}$, respectively, and the capacity $C_{\mathrm{d}, l}$, take into account the deformability of the downstream vessels. The values $P_{l}$ and $\pi_{l}$ are also called proximal and distal pressures, respectively.

Remark 2.1 We will mainly focus on a three-element Windkessel model, that is one of the most popular choices in computational hemodynamics. However, this choice is due purely illustrative and the methods and the analysis presented in the following sections to can be extended to more complex lumped parameter models (see Section 3.2.4 for details).

\subsection{D-0D coupling conditions}

The 3D-0D coupling between (1) and (2) is defined through the following relations on each $\Gamma_{l}$

$$
\left\{\begin{array}{l}
Q_{l}=\int_{\Gamma_{l}} \boldsymbol{u} \cdot \boldsymbol{n}_{\mathrm{f}}, \\
\boldsymbol{\sigma}(\boldsymbol{u}, p) \boldsymbol{n}_{\mathrm{f}}=-P_{l} \boldsymbol{n}_{\mathrm{f}} \text { on } \Gamma_{l},
\end{array}\right.
$$

for $l=1, \ldots, n_{0 \mathrm{D}}$ and where $\boldsymbol{n}_{\mathrm{f}}$ denotes the exterior unit-vector normal of $\Omega_{\mathrm{f}}$.

Energy balance Let the quantity

$$
E(t) \stackrel{\text { def }}{=} \frac{\rho_{\mathrm{f}}}{2}\|\boldsymbol{u}\|_{0, \Omega_{\mathrm{f}}}^{2}+\sum_{l=1}^{n_{0 \mathrm{D}}} \frac{C_{\mathrm{d}, l}}{2} \pi_{l}^{2}
$$

denote the total (kinetic + potential) energy of the 3D-0D coupled system given by $(1)-(3)$, while

$$
D(t) \stackrel{\text { def }}{=} 2 \mu \int_{0}^{t}\|\boldsymbol{\epsilon}(\boldsymbol{u}(s))\|_{0, \Omega_{\mathrm{f}}}^{2} \mathrm{~d} s+\sum_{l=1}^{n_{0 \mathrm{D}}} \int_{0}^{t}\left(\frac{\pi_{l}^{2}(s)}{R_{\mathrm{d}, l}}+R_{\mathrm{p}, l} Q_{l}^{2}(s)\right) \mathrm{d} s>0
$$

represents the dissipative effects. Assuming that $\boldsymbol{u}_{\mathrm{in}}=\mathbf{0}$ (free system) and using a standard energy argument, we get the following identity

$$
E(t)+D(t)+\int_{0}^{t}\left(\int_{\Gamma_{\text {out }}} \frac{\rho_{\mathrm{f}}}{2}|\boldsymbol{u}(s)|^{2} \boldsymbol{u}(s) \cdot \boldsymbol{n}_{\mathrm{f}}\right) \mathrm{d} s=E(0) .
$$


Remark 2.2 Since the last term of the left-hand side of Equation (5) can be negative, this expression does not guarantee a correct energy balance across the 3D-0D interface $\Gamma^{\text {out }}$. This issue is well-known in computational hemodynamics. The interested reader is referred to [4] for a stabilization technique, and to [10] for a different 3D-1D coupling. The methods introduced in this paper can be easily adapted to these alternative formulations.

\subsection{Time semi-discretization}

We consider a fractional-step time-marching of the fluid equations (1) and a backward Euler scheme for the lumped parameter model (2). In what follows, the parameter $\tau$ denotes the time-step size, we set $t_{n} \stackrel{\text { def }}{=} n \tau$ for $n \in \mathbb{N}$ and $\partial_{\tau} x^{n} \stackrel{\text { def }}{=}\left(x^{n}-x^{n-1}\right) / \tau$ stands for the first-order backward difference.

\subsubsection{Fractional-step fluid time-marching}

Several variants of the original Chorin-Temam projection scheme [6, 23] have been proposed in the literature (see, e.g., [15] for a recent review). The methods presented and analyzed in section 3 below do not a priori depend on the specific formulation considered for the projection scheme. To fix the ideas and without generality loss, we consider the non-incremental pressurecorrection version (see, e.g., [15, Section 4]). Hence, the time semi-discrete approximation of 1 is performed as follows. We set $\widetilde{\boldsymbol{u}}^{0}=\boldsymbol{u}^{0}=\boldsymbol{u}_{0}$ and, for $n \geq 1$, we compute $\left(\boldsymbol{u}^{n}, p^{n}, \widetilde{\boldsymbol{u}}^{n}\right)$ by solving: 1. Viscous step:

$$
\left\{\begin{array}{rcc}
\rho_{\mathrm{f}} \frac{\widetilde{\boldsymbol{u}}^{n}-\boldsymbol{u}^{n-1}}{\tau}+\rho_{\mathrm{f}} \widetilde{\boldsymbol{u}}^{n-1} \cdot \boldsymbol{\nabla} \widetilde{\boldsymbol{u}}^{n}-2 \mu \boldsymbol{\nabla} \cdot \boldsymbol{\epsilon}\left(\widetilde{\boldsymbol{u}}^{n}\right)=\mathbf{0} & \text { in } \quad \Omega_{\mathrm{f}} \\
\widetilde{\boldsymbol{u}}^{n}=\boldsymbol{u}_{\mathrm{in}}\left(t_{n}\right) & \text { on } \quad \Gamma^{\text {in }} \\
\widetilde{\boldsymbol{u}}^{n}=\mathbf{0} & \text { on } & \Sigma .
\end{array}\right.
$$

where the 2. Projection step:

$$
\left\{\begin{aligned}
\rho_{\mathrm{f}} \frac{\boldsymbol{u}^{n}-\widetilde{\boldsymbol{u}}^{n}}{\tau}+\boldsymbol{\nabla} p^{n}=\mathbf{0} & \text { in } \quad \Omega_{\mathrm{f}}, \\
\boldsymbol{\nabla} \cdot \boldsymbol{u}^{n}=0 & \text { in } \quad \Omega_{\mathrm{f}}, \\
\boldsymbol{u}^{n} \cdot \boldsymbol{n}_{\mathrm{f}}=\boldsymbol{u}_{\mathrm{in}}\left(t_{n}\right) \cdot \boldsymbol{n}_{\mathrm{f}} & \text { on } \quad \Gamma^{\text {in }}, \\
\boldsymbol{u}^{n}=\mathbf{0} & \text { on } \Sigma .
\end{aligned}\right.
$$

From the implementation point of view, the projection step (7) is usually reformulated by as the pressure-Poisson problem

$$
\left\{\begin{aligned}
-\frac{\tau}{\rho_{\mathrm{f}}} \Delta p^{n}=-\nabla \cdot \widetilde{\boldsymbol{u}}^{n} \quad \text { in } \quad \Omega_{\mathrm{f}} \\
\frac{\tau}{\rho_{\mathrm{f}}} \frac{\partial p^{n}}{\partial \boldsymbol{n}_{\mathrm{f}}}=0 \quad \text { on } \quad \Gamma^{\mathrm{in}} \cup \Sigma,
\end{aligned}\right.
$$

which requires further regularity on the pressure $\left(H^{1}\left(\Omega_{\mathrm{f}}\right)\right.$ instead of $L^{2}\left(\Omega_{\mathrm{f}}\right)$ in practice). Then, the divergence free (or end-of-step) velocity $\boldsymbol{u}^{n}$ can be eliminated in (6) using the following relation (from $\left.(7)_{1}\right)$

$$
\boldsymbol{u}^{n}=\widetilde{\boldsymbol{u}}^{n}-\frac{\tau}{\rho_{\mathrm{f}}} \nabla p^{n} .
$$

It should be noted that the boundary conditions on $\Gamma^{\text {out }}$ have been omitted deliberately in (6)-8), since they depend on the type of 3D-0D coupling scheme considered. In particular, they lead to different formulations of the pressure-Poisson equation (8) (see Section 3.1). 


\subsubsection{Backward-Euler Windkessel time-marching}

Without loss of generality, we consider a backward Euler time-discretization of (2), which yields

$$
\left\{\begin{array}{l}
C_{\mathrm{d}, l} \partial_{\tau} \pi_{l}^{n}+\frac{\pi_{l}^{n}}{R_{\mathrm{d}, l}}=Q_{l}^{n}, \\
P_{l}^{n}=R_{\mathrm{p}, l} Q_{l}^{n}+\pi_{l}^{n}
\end{array}\right.
$$

or, equivalently,

$$
\left\{\begin{array}{l}
\pi_{l}^{n}=\alpha_{l} \pi_{l}^{n-1}+\beta_{l} Q_{l}^{n}, \\
P_{l}^{n}=\gamma_{l} Q_{l}^{n}+\alpha_{l} \pi_{l}^{n-1},
\end{array}\right.
$$

with the notation $\alpha_{l} \stackrel{\text { def }}{=} \frac{R_{\mathrm{d}, l} C_{\mathrm{d}, l}}{R_{\mathrm{d}, l} C_{\mathrm{d}, l}+\tau}, \beta_{l} \stackrel{\text { def }}{=} R_{\mathrm{d}, l}\left(1-\alpha_{l}\right)$ and $\gamma_{l} \stackrel{\text { def }}{=} R_{\mathrm{p}, l}+\beta_{l}$.

\subsection{Spatial discretization}

In what follows, we will consider the usual Sobolev space $H^{1}(\Omega)$, for a given domain $\Omega \subset \mathbb{R}^{3}$. Then, for $X \subset \partial \Omega$ (with meas $(X)>0$ ), we define $H_{X}^{1}(\Omega)$ the subspace of $H^{1}(\Omega)$ with vanishing trace on $X$. The scalar product in $L^{2}(\Omega)$ is denoted by $(\cdot, \cdot)_{\Omega}$ and its associated norm by $\|\cdot\|_{0, \Omega}$.

We consider a family of triangulations $\left\{\mathcal{T}_{\mathrm{f}, h}\right\}_{0<h \leq 1}$ of the domain $\Omega_{\mathrm{f}}$ satisfying the usual requirements of finite element approximations (see, e.g., [7]). The subscript $h \in(0,1]$ refers to the level of refinement of the triangulations. In order to ease the presentation, we assume that the family of triangulations is quasi-uniform. For the discretization in space of (1), we consider continuous Lagrange finite element approximations $\boldsymbol{V}_{h}$ and $R_{h}$ of $\left[H^{1}\left(\Omega_{\mathrm{f}}\right)\right]^{3}$ and $H^{1}\left(\Omega_{\mathrm{f}}\right)$, respectively. Other choices of approximation spaces are possible for the projection method (see [16] for a discussion). For a given $X \subset \partial \Omega_{\mathrm{f}}$ (with meas $(X)>0$ ), we set

$$
\boldsymbol{V}_{X, h} \stackrel{\text { def }}{=} \boldsymbol{V}_{h} \cap\left[H_{X}^{1}\left(\Omega_{\mathrm{f}}\right)\right]^{3}, \quad R_{X, h} \stackrel{\text { def }}{=} R_{h} \cap H_{X}^{1}\left(\Omega_{\mathrm{f}}\right) .
$$

\section{Fractional-step time-marching and 3D-0D coupling schemes}

In this section, we describe two coupling schemes (explicit and implicit) resulting from appropriate time discretizations of the coupling conditions (3).

\subsection{Explicit 3D-0D coupling scheme}

In this case the $3 \mathrm{D}-0 \mathrm{D}$ coupling conditions 3 are time discretized as follows

$$
\left\{\begin{array}{l}
Q_{l}^{n}=\int_{\Gamma_{l}} \widetilde{\boldsymbol{u}}^{n} \cdot \boldsymbol{n}_{\mathrm{f}}, \\
p^{n}=P_{l}^{n} \text { on } \Gamma_{l}, \\
2 \mu \boldsymbol{\epsilon}\left(\widetilde{\boldsymbol{u}}^{n}\right) \boldsymbol{n}_{\mathrm{f}}=\mathbf{0} \text { on } \Gamma_{l},
\end{array}\right.
$$

for $l=1, \ldots, n_{0 \mathrm{D}}$. Note that the continuity of fluxes $\left(3 n_{1}\right.$ is treated explicitly by using the flux of the latest computed viscous velocity. For the relation $(3)_{2}$ we consider a Dirichlet boundary condition for the pressure, while the viscous part of the fluid stresses is set to zero. This is a standard procedure to decouple the projection and viscous steps in the framework of projection schemes with natural boundary conditions (see, e.g., [14] and [15, Section 10]). 
Remark 3.1 It is well-known that the artificial Dirichlet boundary condition $\sqrt{12})_{2}$ could lead to sub-optimal approximations since, in general, the relation $\sqrt{12})_{2}$ is not consistent with the solution of continuous problem. For further details on this issue, we refer to the analysis reported [14] which suggests the use of the rotational version of the pressure-correction scheme.

The resulting fully discrete time-marching procedure is reported in Algorithm 1. In the viscous-step 13) we have considered the standard Temam's consistent term, $\frac{\rho_{\mathrm{f}}}{2}\left(\left(\boldsymbol{\nabla} \cdot \widetilde{\boldsymbol{u}}^{n-1}\right) \widetilde{\boldsymbol{u}}^{n}, \boldsymbol{v}\right)_{\Omega_{\mathrm{f}}}$, which stabilizes the semi-implicit treatment of the convective term. The 3D-0D explicit coupling given by 12 allows a fully uncoupled computation of the Windkessel state, fluid pressure and velocity. This is particularly appealing from the implementation and computational efficiency point of view. Nevertheless, as suggested in Section 3.1.1 below (and then confirmed by numerical experiments in Section 5), Algorithm 1 may suffer from stability issues.

\section{Algorithm 1 (Explicit 3D-0D coupling scheme)}

Let $\boldsymbol{u}^{0} \stackrel{\text { def }}{=} \boldsymbol{u}_{0}, \widetilde{\boldsymbol{u}}^{0} \in \boldsymbol{V}_{h}$ and $\pi_{1}^{0}, \ldots, \pi_{n_{0 \mathrm{D}}}^{0} \in \mathbb{R}$ be given initial data. For $n \geq 1$ perform:

1. Viscous step: Find $\widetilde{\boldsymbol{u}}^{n} \in \boldsymbol{V}_{\Sigma, h}$ such that

$$
\left\{\begin{array}{l}
\left.\widetilde{\boldsymbol{u}}^{n}\right|_{\Gamma^{\mathrm{in}}}=\boldsymbol{u}_{\mathrm{in}}\left(t_{n}\right), \\
\frac{\rho_{\mathrm{f}}}{\tau}\left(\widetilde{\boldsymbol{u}}^{n}, \boldsymbol{v}\right)_{\Omega_{\mathrm{f}}}+\rho_{\mathrm{f}}\left(\widetilde{\boldsymbol{u}}^{n-1} \cdot \nabla \widetilde{\boldsymbol{u}}^{n}, \boldsymbol{v}\right)_{\Omega_{\mathrm{f}}}+\frac{\rho_{\mathrm{f}}}{2}\left(\left(\nabla \cdot \widetilde{\boldsymbol{u}}^{n-1}\right) \widetilde{\boldsymbol{u}}^{n}, \boldsymbol{v}\right)_{\Omega_{\mathrm{f}}} \\
\quad+2 \mu\left(\boldsymbol{\epsilon}\left(\widetilde{\boldsymbol{u}}^{n}\right), \boldsymbol{\epsilon}(\boldsymbol{v})\right)_{\Omega_{\mathrm{f}}}=\frac{\rho_{\mathrm{f}}}{\tau}\left(\boldsymbol{u}^{n-1}, \boldsymbol{v}\right)_{\Omega_{\mathrm{f}}}
\end{array}\right.
$$

for all $\boldsymbol{v} \in \boldsymbol{V}_{\Sigma \cup \Gamma^{\text {in }}, h}$. Thereafter set $\widetilde{Q}_{l}^{n} \stackrel{\text { def }}{=} \int_{\Gamma_{l}} \widetilde{\boldsymbol{u}}^{n} \cdot \boldsymbol{n}_{\mathrm{f}}$.

2. Windkessel step: For $l=1, \ldots, n_{0 \mathrm{D}}$, compute $\left(Q_{l}^{n}, \pi_{l}^{n}, P_{l}^{n}\right) \in \mathbb{R}^{3}$ from

$$
Q_{l}^{n}=\widetilde{Q}_{l}^{n}, \quad C_{\mathrm{d}, l} \partial_{\tau} \pi_{l}^{n}+\frac{\pi_{l}^{n}}{R_{\mathrm{d}, l}}=Q_{l}^{n}, \quad P_{l}^{n}=R_{\mathrm{p}, l} Q_{l}^{n}+\pi_{l}^{n} .
$$

3. Projection step: Find $p^{n} \in R_{h}$ such that

$$
\left\{\begin{array}{l}
\left.p^{n}\right|_{\Gamma_{l}}=P_{l}^{n}, \quad l=1, \ldots, n_{0 \mathrm{D}}, \\
\frac{\tau}{\rho_{\mathrm{f}}}\left(\boldsymbol{\nabla} p^{n}, \nabla q\right)_{\Omega_{\mathrm{f}}}=-\left(\boldsymbol{\nabla} \cdot \widetilde{\boldsymbol{u}}^{n}, q\right)_{\Omega_{\mathrm{f}}}
\end{array}\right.
$$

for all $q \in R_{\Gamma^{\text {out }}, h}$. Thereafter set $\boldsymbol{u}^{n} \stackrel{\text { def }}{=} \widetilde{\boldsymbol{u}}^{n}-\frac{\tau}{\rho_{\mathrm{f}}} \nabla p^{n} \in\left[L^{2}\left(\Omega_{\mathrm{f}}\right)\right]^{d}$.

\subsubsection{Stability analysis}

Let the quantities

$$
\begin{aligned}
& E^{n} \stackrel{\text { def }}{=} \frac{\rho_{\mathrm{f}}}{2}\left\|\widetilde{\boldsymbol{u}}^{n}\right\|_{0, \Omega_{\mathrm{f}}}^{2}+\sum_{l=1}^{n_{0 \mathrm{D}}} \frac{C_{\mathrm{d}, l}}{2}\left|\pi_{l}^{n-1}\right|^{2}, \\
& D^{n} \stackrel{\text { def }}{=} 2 \mu \sum_{m=1}^{n} \tau\left\|\boldsymbol{\epsilon}\left(\tilde{\boldsymbol{u}}^{m}\right)\right\|_{0, \Omega_{\mathrm{f}}}^{2}+\sum_{m=1}^{n-1} \sum_{l=1}^{n_{0 \mathrm{D}}} \tau\left(\frac{\left|\pi_{l}^{m}\right|^{2}}{R_{\mathrm{d}, l}}+R_{\mathrm{p}, l}\left|Q_{l}^{m}\right|^{2}\right),
\end{aligned}
$$

for $n \geq 1$, denote the energy and physical dissipation of the discrete system. Let us also set

$$
E^{0} \stackrel{\text { def }}{=} \frac{\rho_{\mathrm{f}}}{2}\left\|\boldsymbol{u}^{0}\right\|_{0, \Omega_{\mathrm{f}}}^{2}+\sum_{l=1}^{n_{0 \mathrm{D}}} \frac{C_{\mathrm{d}, l}}{2}\left|\pi_{l}^{0}\right|^{2},
$$


We then have the following energy based result (whose proof can be found in [5, Chapter 2]).

Theorem 3.2 Let $\left\{\left(\widetilde{\boldsymbol{u}}^{n}, p^{n}\right)\right\}_{n>1}$ and $\left\{\left(Q_{l}^{n}, \pi_{l}^{n}, P_{l}^{n}\right)_{1 \leq l \leq n_{0 \mathrm{D}}}\right\}_{n>1}$ be the solution given by Algorithm 1 and assume that $\boldsymbol{u}_{\mathrm{in}}=\mathbf{0}$ (free system). The following inequality holds for $n \geq 1$

$$
\begin{aligned}
E^{n}+D^{n}+\sum_{m=1}^{n} \frac{\rho_{\mathrm{f}}}{2} \tau\left(\widetilde{\boldsymbol{u}}^{m-1} \cdot \boldsymbol{n}_{\mathrm{f}},\left|\widetilde{\boldsymbol{u}}^{m}\right|^{2}\right)_{\Gamma \text { out }} \leq E^{0}-\sum_{m=1}^{n-1} \frac{\tau^{2}}{2 \rho_{\mathrm{f}}}\left\|\boldsymbol{\nabla} p^{m}\right\|_{0, \Omega_{\mathrm{f}}}^{2} \\
+\sum_{m=1}^{n-1} \tau\left(\boldsymbol{\nabla} \cdot \widetilde{\boldsymbol{u}}^{m}, \phi^{m}\right)_{\Omega_{\mathrm{f}}}+\sum_{m=1}^{n-1} \frac{\tau^{2}}{\rho_{\mathrm{f}}}\left(\boldsymbol{\nabla} p^{m}, \boldsymbol{\nabla} \phi^{m}\right)_{\Omega_{\mathrm{f}}},
\end{aligned}
$$

where $\phi^{n} \in R_{h}$ is an arbitrary discrete lifting of the (unknown) proximal pressures, namely,

$$
\phi^{n}=P_{l}^{n} \quad \text { on } \quad \Gamma_{l}, l=1, \ldots, n_{0 \mathrm{D}} .
$$

The left-hand side of estimate (16) corresponds to the discrete counterpart of (5). Nevertheless, the artificial power introduced by the last two terms of 16 ,

$$
\sum_{m=1}^{n-1} \tau\left(\boldsymbol{\nabla} \cdot \widetilde{\boldsymbol{u}}^{m}, \phi^{m}\right)_{\Omega_{\mathrm{f}}}+\sum_{m=1}^{n-1} \frac{\tau^{2}}{\rho_{\mathrm{f}}}\left(\boldsymbol{\nabla} p^{m}, \boldsymbol{\nabla} \phi^{m}\right)_{\Omega_{\mathrm{f}}},
$$

cannot be controlled, so that this estimate does not guarantee the energy stability of the approximations provided by Algorithm 1. Two remarks are now in order.

Remark 3.3 It is worth mentioning that the term (18) corresponds to the residual of the projection step (15) (note that $\phi^{n} \notin R_{\Gamma^{\text {out }}, h}$ ). In fact, for the space continuous counterpart of 15 we have

$$
\begin{aligned}
\left(\boldsymbol{\nabla} \cdot \widetilde{\boldsymbol{u}}^{n}, \phi^{n}\right)_{\Omega_{\mathrm{f}}}+\frac{\tau}{\rho_{\mathrm{f}}}\left(\boldsymbol{\nabla} p^{n}, \boldsymbol{\nabla} \phi^{n}\right)_{\Omega_{\mathrm{f}}} & =\frac{\tau}{\rho_{\mathrm{f}}} \int_{\Gamma^{\mathrm{out}}} \frac{\partial p^{n}}{\partial \boldsymbol{n}_{\mathrm{f}}} \phi^{n} \\
& =-\sum_{l=1}^{n_{\mathrm{OD}}}\left(\int_{\Gamma_{l}} \boldsymbol{u}^{n} \cdot \boldsymbol{n}_{\mathrm{f}}-\int_{\Gamma_{l}} \widetilde{\boldsymbol{u}}^{n} \cdot \boldsymbol{n}_{\mathrm{f}}\right) P_{l}^{n} .
\end{aligned}
$$

Hence, the uncontrolled artificial power involved in the energy estimate 16 is due to the time-lag in the flux $\left(\int_{\Gamma_{l}} \widetilde{\boldsymbol{u}}^{n} \cdot \boldsymbol{n}_{\mathrm{f}}\right.$ instead of $\left.\int_{\Gamma_{l}} \boldsymbol{u}^{n} \cdot \boldsymbol{n}_{\mathrm{f}}\right)$ introduced by the explicit treatment of the continuity of fluxes 12 on the 3D-OD interfaces $\Gamma_{l}$. It should be noted that a 3D-OD explicit coupling with a monolithic time-marching scheme in the fluid yields a similar time lag, but now with $\int_{\Gamma_{l}} \boldsymbol{u}^{n-1} \cdot \boldsymbol{n}$ instead of $\int_{\Gamma_{l}} \boldsymbol{u}^{n} \cdot \boldsymbol{n}$.

Remark 3.4 In the case of a single outlet (i.e., $n_{0 \mathrm{D}}=1$ ), we can take $\phi^{n}=P^{n}$ in $\Omega_{\mathrm{f}}$. From the proof of Proposition 3.2 (see [5, Chapter 2]) one then recovers the following energy estimate for the fluid

$$
\begin{aligned}
& \frac{\rho_{\mathrm{f}}}{2}\left\|\widetilde{\boldsymbol{u}}^{n}\right\|_{0, \Omega_{\mathrm{f}}}^{2}+2 \mu \sum_{m=1}^{n} \tau\left\|\boldsymbol{\epsilon}\left(\tilde{\boldsymbol{u}}^{m}\right)\right\|_{0, \Omega_{\mathrm{f}}}^{2}+\sum_{m=1}^{n} \frac{\rho_{\mathrm{f}}}{2} \tau\left(\widetilde{\boldsymbol{u}}^{m-1} \cdot \boldsymbol{n}_{\mathrm{f}},\left|\widetilde{\boldsymbol{u}}^{m}\right|^{2}\right)_{\Gamma} \text { out } \\
& \leq \frac{\rho_{\mathrm{f}}}{2}\left\|\boldsymbol{u}^{0}\right\|_{0, \Omega_{\mathrm{f}}}^{2}-\sum_{m=1}^{n-1} \frac{\tau^{2}}{2 \rho_{\mathrm{f}}}\left\|\boldsymbol{\nabla} p^{m}\right\|_{0, \Omega_{\mathrm{f}}}^{2} .
\end{aligned}
$$

As a result, the energy stability of the fluid does not depend on the imposed outlet pressure. 


\subsection{Implicit 3D-0D coupling scheme}

In this case the 3D-0D coupling conditions (3) are time-discretized as follows

$$
\left\{\begin{array}{l}
Q_{l}^{n}=\int_{\Gamma_{l}} \boldsymbol{u}^{n} \cdot \boldsymbol{n}_{\mathrm{f}}, \\
p^{n}=P_{l}^{n} \text { on } \Gamma_{l}, \\
2 \mu \boldsymbol{\epsilon}\left(\widetilde{\boldsymbol{u}}^{n}\right) \boldsymbol{n}_{\mathrm{f}}=\mathbf{0} \text { on } \Gamma_{l},
\end{array}\right.
$$

for $l=1, \ldots, n_{0 \mathrm{D}}$. Note that, in comparison with $\sqrt{12}$, the above coupling scheme treats implicitly the continuity of fluxes on the outlet boundaries. This feature enhances stability, as we will show in Section 3.2.1. However, from the computational point of view, the relations $(19)_{1,2}$ and (10) apparently couple the evaluation of (8) and (9). Fortunately, this difficulty can be circumvented via an appropriate reformulation of the pressure boundary condition $(19)_{2}$ for the projection step (8). Indeed, by inserting $(9)$ into $(19)_{1}$, we get

$$
Q_{l}^{n}=\widetilde{Q}_{l}^{n}-\frac{\tau}{\rho_{\mathrm{f}}} \int_{\Gamma_{l}} \frac{\partial p^{n}}{\partial \boldsymbol{n}_{\mathrm{f}}},
$$

which with 11 and $(19)_{2}$ yields the following (implicit) boundary condition for the outlet pressures:

$$
\left.p^{n}\right|_{\Gamma_{l}}=\gamma_{l} \widetilde{Q}_{l}^{n}-\frac{\gamma_{l} \tau}{\rho_{\mathrm{f}}} \int_{\Gamma_{l}} \frac{\partial p^{n}}{\partial \boldsymbol{n}_{\mathrm{f}}}+\alpha_{l} \pi_{l}^{n-1}
$$

for $l=1, \ldots, n_{0 \mathrm{D}}$. Note that this expression still enforces $p^{n}$ to be constant on each $\Gamma_{l}$.

Multiplying $(8)_{1}$ by $q \in R_{h}$, integrating by parts, using $(8)_{1}$ and the fact that $\left.q\right|_{\Gamma_{l}}$ is constant, we get

$$
\frac{\tau}{\rho_{\mathrm{f}}}\left(\boldsymbol{\nabla} p^{n}, \boldsymbol{\nabla} q\right)_{\Omega_{\mathrm{f}}}-\left.\frac{\tau}{\rho_{\mathrm{f}}} \sum_{l=1}^{n_{0 \mathrm{D}}}\left(\int_{\Gamma_{l}} \frac{\partial p^{n}}{\partial \boldsymbol{n}_{\mathrm{f}}}\right) q\right|_{\Gamma_{l}}=-\left(\boldsymbol{\nabla} \cdot \widetilde{\boldsymbol{u}}^{n}, q\right)_{\Omega_{\mathrm{f}}}
$$

for all $q \in R_{h}$. We can eliminate the normal derivative of the pressure using (21), which yields the following modified variational formulation for the projection step: Find $p^{n} \in R_{h}$ such that

$$
\frac{\tau}{\rho_{\mathrm{f}}}\left(\nabla p^{n}, \boldsymbol{\nabla} q\right)_{\Omega_{\mathrm{f}}}+\sum_{l=1}^{n_{0 \mathrm{D}}} \frac{\left(\left.p^{n}\right|_{\Gamma_{l}}\right)\left(\left.q\right|_{\Gamma_{l}}\right)}{\gamma_{l}}=\left.\sum_{l=1}^{n_{0 \mathrm{D}}}\left(\widetilde{Q}_{l}^{n}+\frac{\alpha_{l} \pi_{l}^{n-1}}{\gamma_{l}}\right) q\right|_{\Gamma_{l}}-\left(\boldsymbol{\nabla} \cdot \widetilde{\boldsymbol{u}}^{n}, q\right)_{\Omega_{\mathrm{f}}}
$$

for all $q \in R_{h}$. We can then set $P_{l}^{n}=\left.p^{n}\right|_{\Gamma_{l}}$ and retreive $\left(Q_{l}^{n}, \pi_{l}^{n}\right)$ from $\left[11\right.$, for $l=1, \ldots n_{0 \mathrm{D}}$.

Remark 3.5 The well-posedness of the pressure-Poisson problem 22) follows from a generalized Poincaré's inequality, which guarantees the coercivity of the left-hand side of (22) in $R_{h}$.

Remark 3.6 Testing 22 with $q=1$, and since $P_{l}^{n}=\left.p^{n}\right|_{\Gamma_{l}}$, we have

$$
\sum_{l=1}^{n_{0 \mathrm{D}}} \frac{P_{l}^{n}-\alpha_{l} \pi_{l}^{n-1}}{\gamma_{l}}=\sum_{l=1}^{n_{0 \mathrm{D}}} \widetilde{Q}_{l}^{n}+\int_{\Omega_{\mathrm{f}}} \boldsymbol{\nabla} \cdot \widetilde{\boldsymbol{u}}^{n}
$$

Hence, integrating by parts in the last term, using $[11]_{2}$ and owing to 6$]_{2,3}$ we get the following mass conservation for the Windkessel fluxes:

$$
\sum_{l=1}^{n_{0 \mathrm{D}}} Q_{l}^{n}=-\int_{\Gamma^{\mathrm{in}}} \boldsymbol{u}_{\mathrm{in}}\left(t_{n}\right) .
$$


The complete time-marching procedures is reported in Algorithm 2.

\section{Algorithm 2 (3D-0D implicit coupling scheme)}

Let $\boldsymbol{u}^{0}=\boldsymbol{u}_{0}, \widetilde{\boldsymbol{u}}^{0} \in \boldsymbol{V}_{h}$, and $\pi_{1}^{0}, \ldots, \pi_{n_{0 \mathrm{D}}}^{0} \in \mathbb{R}$ be given initial data. For $n \geq 1$ perform: 1. Viscous step: Find $\widetilde{\boldsymbol{u}}^{n} \in \boldsymbol{V}_{\Sigma, h}$ such that

$$
\left\{\begin{array}{l}
\left.\widetilde{\boldsymbol{u}}^{n}\right|_{\Gamma^{\mathrm{in}}}=\boldsymbol{u}_{\mathrm{in}}\left(t_{n}\right) \\
\frac{\rho_{\mathrm{f}}}{\tau}\left(\widetilde{\boldsymbol{u}}^{n}, \boldsymbol{v}\right)_{\Omega_{\mathrm{f}}}+\rho_{\mathrm{f}}\left(\widetilde{\boldsymbol{u}}^{n-1} \cdot \nabla \widetilde{\boldsymbol{u}}^{n}, \boldsymbol{v}\right)_{\Omega_{\mathrm{f}}}+2 \mu\left(\boldsymbol{\epsilon}\left(\widetilde{\boldsymbol{u}}^{n}\right), \boldsymbol{\epsilon}(\boldsymbol{v})\right)_{\Omega_{\mathrm{f}}} \\
\quad+=\frac{\rho_{\mathrm{f}}}{\tau}\left(\boldsymbol{u}^{n-1}, \boldsymbol{v}\right)_{\Omega_{\mathrm{f}}}
\end{array}\right.
$$

for all $\boldsymbol{v} \in \boldsymbol{V}_{\Sigma \cup \Gamma^{\text {in }}, h}$. Thereafter set $\widetilde{Q}_{l}^{n} \stackrel{\text { def }}{=} \int_{\Gamma_{l}} \widetilde{\boldsymbol{u}}^{n} \cdot \boldsymbol{n}_{\mathrm{f}}$.

2. Projection-Windkessel step: Find $p^{n} \in R_{h}$ and such that

$$
\frac{\tau}{\rho_{\mathrm{f}}}\left(\boldsymbol{\nabla} p^{n}, \boldsymbol{\nabla} q\right)_{\Omega_{\mathrm{f}}}+\sum_{l=1}^{n_{0 \mathrm{D}}} \frac{\left(\left.p^{n}\right|_{\Gamma_{l}}\right)\left(\left.q\right|_{\Gamma_{l}}\right)}{\gamma_{l}}=\left.\sum_{l=1}^{n_{0 \mathrm{D}}}\left(\widetilde{Q}_{l}^{n}+\frac{\alpha_{l} \pi_{l}^{n-1}}{\gamma_{l}}\right) q\right|_{\Gamma_{l}}-\left(\boldsymbol{\nabla} \cdot \widetilde{\boldsymbol{u}}^{n}, q\right)_{\Omega_{\mathrm{f}}}
$$

for all $q \in R_{h}$. Thereafter, set $P_{l}^{n}=\left.p^{n}\right|_{\Gamma_{l}}$ and compute $\left(Q_{l}^{n}, \pi_{l}^{n}\right) \in \mathbb{R}^{2}$ from the relations

$$
Q_{l}^{n}=\frac{P_{l}^{n}-\alpha_{l} \pi_{l}^{n-1}}{\gamma_{l}}, \quad \pi_{l}^{n}=\alpha_{l} \pi_{l}^{n-1}+\beta_{l} Q_{l}^{n}, \quad l=1, \ldots n_{0 \mathrm{D}}
$$

and set $\boldsymbol{u}^{n} \stackrel{\text { def }}{=} \widetilde{\boldsymbol{u}}^{n}-\frac{\tau}{\rho_{\mathrm{f}}} \nabla p^{n} \in\left[L^{2}\left(\Omega_{\mathrm{f}}\right)\right]^{d}$.

\subsubsection{Stability analysis}

The focus of this section is to present the stability result of the formulation (23)- 25 summarized in the following proposition.

Theorem 3.7 Let $\left\{\left(\widetilde{\boldsymbol{u}}^{n}, p^{n}\right)\right\}_{n \geq 1}$ and $\left\{\left(Q_{l}^{n}, \pi_{l}^{n}, P_{l}^{n}\right)_{1 \leq l \leq n_{0 \mathrm{D}}}\right\}_{n>1}$ be the approximated solution given by Algorithm 2 and assume that $\boldsymbol{u}_{\mathrm{in}}=\mathbf{0}$ (free system). The following energy inequality holds

$$
E^{n}+D^{n}+\sum_{m=1}^{n} \frac{\rho_{\mathrm{f}}}{2}\left(\widetilde{\boldsymbol{u}}^{m-1} \cdot \boldsymbol{n}_{\mathrm{f}},\left|\widetilde{\boldsymbol{u}}^{m}\right|^{2}\right)_{\Gamma^{\text {out }}} \leq E^{0}-\sum_{m=1}^{n-1} \frac{\tau^{2}}{2 \rho_{\mathrm{f}}}\left\|\nabla p^{m}\right\|_{0, \Omega_{\mathrm{f}}}^{2} .
$$

Proof. We first test 23 with $\boldsymbol{v}=\widetilde{\boldsymbol{u}}^{n}$ and integrate by parts the convective term. This yields the identity

$$
\begin{aligned}
\frac{\rho_{\mathrm{f}}}{2} \partial_{\tau}\left\|\widetilde{\boldsymbol{u}}^{n}\right\|_{0, \Omega_{\mathrm{f}}}^{2}+\frac{\rho_{\mathrm{f}}}{2 \tau}\left\|\widetilde{\boldsymbol{u}}^{n}-\widetilde{\boldsymbol{u}}^{n-1}\right\|_{0, \Omega_{\mathrm{f}}}^{2}+ & 2 \mu\left\|\boldsymbol{\epsilon}\left(\widetilde{\boldsymbol{u}}^{n}\right)\right\|_{0, \Omega_{\mathrm{f}}}^{2} \\
& +\left(\boldsymbol{\nabla} p^{n-1}, \widetilde{\boldsymbol{u}}^{n}\right)_{\Omega_{\mathrm{f}}}+\frac{\rho_{\mathrm{f}}}{2}\left(\widetilde{\boldsymbol{u}}^{n-1} \cdot \boldsymbol{n}_{\mathrm{f}},\left|\widetilde{\boldsymbol{u}}^{n}\right|^{2}\right)_{\Gamma \text { out }}=0
\end{aligned}
$$

for $n \geq 2$ and, for $n=1$, we get

$$
\frac{\rho_{\mathrm{f}}}{2 \tau}\left(\left\|\widetilde{\boldsymbol{u}}^{1}\right\|_{0, \Omega_{\mathrm{f}}}^{2}-\left\|\boldsymbol{u}^{0}\right\|_{0, \Omega_{\mathrm{f}}}^{2}\right)+\frac{\rho_{\mathrm{f}}}{2 \tau}\left\|\widetilde{\boldsymbol{u}}^{1}-\boldsymbol{u}^{0}\right\|_{0, \Omega_{\mathrm{f}}}^{2}+2 \mu\left\|\boldsymbol{\epsilon}\left(\widetilde{\boldsymbol{u}}^{1}\right)\right\|_{0, \Omega_{\mathrm{f}}}^{2}+\frac{\rho_{\mathrm{f}}}{2}\left(\widetilde{\boldsymbol{u}}^{0} \cdot \boldsymbol{n}_{\mathrm{f}},\left|\widetilde{\boldsymbol{u}}^{1}\right|^{2}\right)_{\Gamma^{\text {out }}}=0 .
$$


Thereafter, taking (24) at time step $n-1$, testing with $q=p^{n-1}$ and integrating by parts in its right-hand side it yields

$$
\frac{\tau}{\rho_{\mathrm{f}}}\left\|\nabla p^{n-1}\right\|_{0, \Omega_{\mathrm{f}}}^{2}+\sum_{l=1}^{n_{0 \mathrm{D}}} \frac{P_{l}^{n-1}-\alpha_{l} \pi_{l}^{n-2}}{\gamma_{l}} P_{l}^{n-1}=\left(\widetilde{\boldsymbol{u}}^{n-1}, \nabla p^{n-1}\right)_{\Omega_{\mathrm{f}}} .
$$

for $n \geq 2$. Hence, the addition and subtraction of suitable terms and the application of the Cauchy-Schwarz and arithmetic-geometric inequalities yields

$$
\frac{\tau}{2 \rho_{\mathrm{f}}}\left\|\boldsymbol{\nabla} p^{n-1}\right\|_{0, \Omega_{\mathrm{f}}}^{2}-\frac{\rho_{\mathrm{f}}}{2 \tau}\left\|\widetilde{\boldsymbol{u}}^{n}-\widetilde{\boldsymbol{u}}^{n-1}\right\|_{0, \Omega_{\mathrm{f}}}^{2}-\left(\widetilde{\boldsymbol{u}}^{n}, \boldsymbol{\nabla} p^{n-1}\right)_{\Omega_{\mathrm{f}}}+\sum_{l=1}^{n_{0 \mathrm{D}}} \frac{P_{l}^{n-1}-\alpha_{l} \pi_{l}^{n-2}}{\gamma_{l}} P_{l}^{n-1} \leq 0 .
$$

As a result, the summation of 27 and $(29)$ gives

$$
\begin{aligned}
\frac{\rho_{\mathrm{f}}}{2} \partial_{\tau}\left\|\widetilde{\boldsymbol{u}}^{n}\right\|_{0, \Omega_{\mathrm{f}}}^{2}+2 \mu\left\|\boldsymbol{\epsilon}\left(\widetilde{\boldsymbol{u}}^{n}\right)\right\|_{0, \Omega_{\mathrm{f}}}^{2}+\frac{\rho_{\mathrm{f}}}{2}\left(\widetilde{\boldsymbol{u}}^{n-1} \cdot \boldsymbol{n}_{\mathrm{f}},\left|\widetilde{\boldsymbol{u}}^{n}\right|^{2}\right)_{\Gamma} \text { out } \\
\quad+\sum_{l=1}^{n_{0 \mathrm{D}}} \frac{P_{l}^{n-1}-\alpha_{l} \pi_{l}^{n-2}}{\gamma_{l}} P_{l}^{n-1} \leq-\frac{\tau}{2 \rho_{\mathrm{f}}}\left\|\nabla p^{n-1}\right\|_{0, \Omega_{\mathrm{f}}}^{2}
\end{aligned}
$$

for $n \geq 2$. At last, from $[25$ and its equivalence to $(11)$, we have

$$
\begin{aligned}
\frac{P_{l}^{n}-\alpha_{l} \pi_{l}^{n-1}}{\gamma_{l}} P_{l}^{n} & =Q_{l}^{n} P_{l}^{n}=R_{\mathrm{p}, l}\left|Q_{l}^{n}\right|^{2}+Q_{l}^{n} \pi_{l}^{n} \\
& \geq \frac{C_{\mathrm{d}, l}}{2} \partial_{\tau}\left|\pi_{l}^{n}\right|^{2}+\frac{1}{R_{\mathrm{d}, l}}\left|\pi_{l}^{n}\right|^{2}+R_{\mathrm{p}, l}\left|Q_{l}^{n}\right|^{2}
\end{aligned}
$$

for $n \geq 1$. Hence, by inserting the last inequality of (31) into $(30)$, multiplying by $\tau$ and summing over $m=2, \ldots, n$ we get the estimate

$$
E^{n}+D^{n}+\sum_{m=2}^{n} \frac{\rho_{\mathrm{f}}}{2}\left(\widetilde{\boldsymbol{u}}^{m-1} \cdot \boldsymbol{n}_{\mathrm{f}},\left|\widetilde{\boldsymbol{u}}^{m}\right|^{2}\right)_{\Gamma^{\mathrm{out}}} \leq E^{1}-\sum_{m=1}^{n-1} \frac{\tau^{2}}{2 \rho_{\mathrm{f}}}\left\|\nabla p^{m}\right\|_{0, \Omega_{\mathrm{f}}}^{2} .
$$

for $n \geq 2$. We recover the estimate 26 by simply adding to this inequality the expression 28 multiplied by $\tau$, which completes the proof.

The estimate (26) corresponds to the discrete counterpart of (5). Note that the right-hand side of 26 is a pure numerical dissipation term (the natural pressure stabilization of the projection scheme). Therefore, the 3D-0D coupling reported in Algorithm 2 does not introduce any uncontrolled artificial power and, hence, a guaranty of numerical stability. This feature will be illustrated in Section 5 via numerical experiments.

\subsubsection{Implementation details}

In this section we discuss the implementation of the pressure problem 24 in a finite element framework. For the sake of simplicity, and without loss of generality, we limit the discussion to the case of a single outlet. We define the arrays $P, V \in \mathbb{R}^{N}$ corresponding to the degrees of freedom (d.o.f.) of the pressure $p^{n} \in Q_{h}$ and of a general test function $q \in Q_{h}$, respectively. The bilinear form $\left(\nabla p^{n}, \nabla q\right)_{\Omega_{\mathrm{f}}}$, without imposing Dirichlet boundary conditions to $p^{n}$, can be written in matrix form as

$$
\left(\nabla p^{n}, \nabla q\right)_{\Omega_{\mathrm{f}}}=V^{T} A P=\left[\begin{array}{ll}
V_{I}^{T} & V_{O}^{T}
\end{array}\right]\left[\begin{array}{ll}
A_{I I} & A_{I O} \\
A_{O I} & A_{O O}
\end{array}\right]\left[\begin{array}{c}
P_{I} \\
P_{O}
\end{array}\right]
$$


where the subindexes $O$ and $I$ indicate the elements of the array corresponding to the d.o.f. on $\Gamma^{\text {out }}$ and $\bar{\Omega} \backslash \Gamma^{\text {out }}$, respectively. Hence, the pressure-Poisson projection step with explicit Dirichlet data can be formulated as

$$
A_{I I} P_{I}=\widetilde{F}_{I}-A_{I O} \mathbb{1}_{O} p_{\mid \Gamma^{\text {out }}}^{n},
$$

where the notation in right-hand side is such that

$$
\left[\begin{array}{ll}
V_{I}^{T} & V_{O}^{T}
\end{array}\right]\left[\begin{array}{c}
\widetilde{F}_{I} \\
\widetilde{F}_{O}
\end{array}\right]=\frac{\rho_{\mathrm{f}}}{\tau}\left(\nabla \cdot \widetilde{\boldsymbol{u}}^{n}, q\right)_{\Omega_{\mathrm{f}}}
$$

with $\mathbb{1}_{O} \in \mathbb{R}^{N_{O}}$ denoting a vector of ones, with $N_{O}$ being the number of pressure d.o.f. on $\Gamma^{\text {out }}$. The linear system 32 is usually solved by means of a preconditioned conjugate gradient methods (PCG), with the preconditioning operator $\widehat{A}_{I I}^{-1}$ given, for example, by an incomplete Cholesky factorization of $A_{I I}$ (alternative preconditioners could be used). With the notations introduced above, the stiffness matrix of the implicit formulation (24) can be derived straightforwardly. Indeed, since $V_{O}=\mathbb{1}_{O q_{\mid \Gamma^{\text {out }}}}$ and $P_{O}=\mathbb{1}_{O} p_{\mid \Gamma_{\text {out }}}^{n}$, we obtain

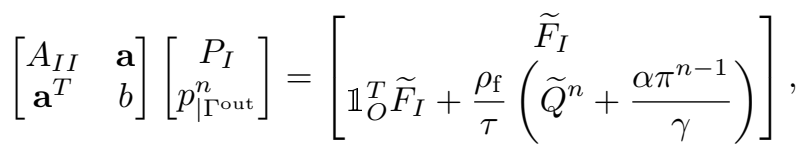

with $\mathbf{a} \stackrel{\text { def }}{=} A_{I O} \mathbb{1}_{O}$ and $b \stackrel{\text { def }}{=} \mathbb{1}_{O}^{T} A_{O O} \mathbb{1}_{O}+\frac{\rho_{\mathrm{f}}}{\tau \gamma}$. It should be noted that the matrix in (33) has a non-standard sparsity pattern. However, since we use a Krylov linear solver this matrix is never assembled in practice (only matrix-vector products are evaluated). In the numerical experiments, we have considered the block-preconditioner given by

$$
\left[\begin{array}{cc}
\widehat{A}_{I I}^{-1} & 0 \\
0 & \frac{1}{b}
\end{array}\right]
$$

which yielded practically the same number of PCG-iterations than in the solution of $(32)$. In the general case of a domain $\Omega_{\mathrm{f}}$ with multiple outlets, the aforementioned considerations can be extended by considering one additional equation for each 3D-0D interface. Concerning the computational cost, in our numerical simulations we did observe any relevant difference between the implicit and the explicit coupling.

\subsubsection{Extension to higher order time-splitting schemes}

Although widely used in practice, the original Chorin-Temam projection scheme might suffer of a limited accuracy in time and of spurious boundary layers, due to the unphysical homogenous Neumann boundary condition $[8]_{3}$. Among the several variants available (see, e.g., [15] for an overview), in this section we describe a possible extension of the implicit 3D-0D coupling (Algorithm 2) in the context of an incremental pressure projection scheme with a second order time discretization.

Following [15], we decompose now the time iteration in a BDF2 time discretization for the viscous step

$\left\{\begin{array}{rll}\rho_{\mathrm{f}} \frac{\left(3 \widetilde{\boldsymbol{u}}^{n}-4 \boldsymbol{u}^{n-1}+\boldsymbol{u}^{n-2}\right)}{2 \tau}+\rho_{\mathrm{f}} \widetilde{\boldsymbol{u}}^{n-1} \cdot \boldsymbol{\nabla} \widetilde{\boldsymbol{u}}^{n}-2 \mu \boldsymbol{\nabla} \cdot \boldsymbol{\epsilon}\left(\widetilde{\boldsymbol{u}}^{n}\right)+\nabla p^{n-1}=\mathbf{0} & \text { in } \quad \Omega_{\mathrm{f}}, \\ \mathrm{RR} \mathrm{n}^{\circ} & \widetilde{\boldsymbol{u}}^{n}=\boldsymbol{u}_{\mathrm{in}}\left(t_{n}\right) & \text { on } \quad \Gamma^{\text {in }}, \\ \widetilde{\boldsymbol{u}}^{n}=\mathbf{0} & \text { on } & \Sigma, \\ 2 \mu \boldsymbol{\epsilon}\left(\widetilde{\boldsymbol{u}}^{n}\right) \boldsymbol{n}_{\mathrm{f}}=\mathbf{0} & \text { on } \quad \Gamma^{\text {out }},\end{array}\right.$ 
a projection step for the increment of pressure

$$
\left\{\begin{aligned}
&-\frac{\tau}{\rho_{\mathrm{f}}} \Delta \delta p^{n}=-\frac{3}{2} \nabla \cdot \widetilde{\boldsymbol{u}}^{n} \quad \text { in } \quad \Omega_{\mathrm{f}}, \\
& \frac{\tau}{\rho_{\mathrm{f}}} \frac{\partial \delta p^{n}}{\partial \boldsymbol{n}_{\mathrm{f}}}=0 \quad \text { on } \quad \Gamma^{\mathrm{in}} \cup \Sigma, \\
& \delta p^{n}=P_{l}^{n}-P_{l}^{n-1} \quad \text { on } \quad \Gamma_{l}, \quad l=1, \ldots, n_{0 \mathrm{D}}
\end{aligned}\right.
$$

completed by the end-of-step updates: $p^{n}=p^{n-1}+\delta p^{n}$ and $\boldsymbol{u}^{n}=\widetilde{\boldsymbol{u}}^{n}-\frac{2 \tau}{3 \rho_{\mathrm{f}}} \boldsymbol{\nabla} \delta p^{n}$.

In order to have a second-order time-discretization for the whole $3 \mathrm{D}-0 \mathrm{D}$ problem, we also discretize the 0D model with a BDF2 scheme, namely

$$
\pi_{l}^{n}=\widehat{\alpha}_{l} \pi_{l}^{n-1}-\frac{\widehat{\alpha}_{l}}{4} \pi_{l}^{n-2}+\widehat{\beta}_{l} Q_{l}^{n}, \quad P_{l}^{n}=\widehat{\gamma}_{l} Q_{l}^{n}+\widehat{\alpha}_{l} \pi_{l}^{n-1}-\frac{\widehat{\alpha}_{l}}{4} \pi_{l}^{n-2},
$$

with $\widehat{\alpha}_{l} \stackrel{\text { def }}{=} \frac{2 R_{\mathrm{d}, l} C_{\mathrm{d}, l}}{(3 / 2) R_{\mathrm{d}, l} C_{\mathrm{d}, l}+\tau}, \widehat{\beta}_{l} \stackrel{\text { def }}{=} \frac{R_{\mathrm{d}, l} \tau}{(3 / 2) R_{\mathrm{d}, l} C_{\mathrm{d}, l}+\tau}, \widehat{\gamma}_{l} \stackrel{\text { def }}{=} R_{\mathrm{p}, l}+\widehat{\beta}_{l}$.

Hence, using equation 35$]_{2}$ to define the implicit coupling with the projection step, we obtain the following time-stepping method:

1. Viscous step: Find $\widetilde{\boldsymbol{u}}^{n} \in \boldsymbol{V}_{\Sigma, h}$ such that

$$
\left\{\begin{array}{l}
\left.\widetilde{\boldsymbol{u}}^{n}\right|_{\Gamma^{\text {in }}}=\boldsymbol{u}_{\text {in }}\left(t_{n}\right), \\
\frac{\rho_{\mathrm{f}}}{2 \tau}\left(3 \widetilde{\boldsymbol{u}}^{n}, \boldsymbol{v}\right)_{\Omega_{\mathrm{f}}}+\rho_{\mathrm{f}}\left(\widetilde{\boldsymbol{u}}^{n-1} \cdot \nabla \widetilde{\boldsymbol{u}}^{n}, \boldsymbol{v}\right)_{\Omega_{\mathrm{f}}}+2 \mu\left(\boldsymbol{\epsilon}\left(\widetilde{\boldsymbol{u}}^{n}\right), \boldsymbol{\epsilon}(\boldsymbol{v})\right)_{\Omega_{\mathrm{f}}} \\
\quad+\frac{\rho_{\mathrm{f}}}{2}\left(\left(\boldsymbol{\nabla} \cdot \widetilde{\boldsymbol{u}}^{n-1}\right) \widetilde{\boldsymbol{u}}^{n}, \boldsymbol{v}\right)_{\Omega_{\mathrm{f}}}=-\left(\boldsymbol{\nabla} p^{n}, \boldsymbol{v}\right)+\frac{\rho_{\mathrm{f}}}{2 \tau}\left(4 \boldsymbol{u}^{n-1}-\boldsymbol{u}^{n-2}, \boldsymbol{v}\right)_{\Omega_{\mathrm{f}}}
\end{array}\right.
$$

for all $\boldsymbol{v} \in \boldsymbol{V}_{\Sigma \cup \Gamma^{\text {in }}, h}$. Thereafter set $\widetilde{Q}_{l}^{n} \stackrel{\text { def }}{=} \int_{\Gamma_{l}} \widetilde{\boldsymbol{u}}^{n} \cdot \boldsymbol{n}_{\mathrm{f}}$.

2. Increment-Windkessel step: Find $\delta p^{n} \in R_{h}$ and such that

$$
\begin{aligned}
\frac{\tau}{\rho_{\mathrm{f}}}\left(\boldsymbol{\nabla} \delta p^{n}, \boldsymbol{\nabla} q\right)_{\Omega_{\mathrm{f}}}+\sum_{l=1}^{n_{0 \mathrm{D}}} \frac{\left(\left.\delta p^{n}\right|_{\Gamma_{l}}\right)\left(\left.q\right|_{\Gamma_{l}}\right)}{\widehat{\gamma}_{l}} & \\
& =\left.\sum_{l=1}^{n_{0 \mathrm{D}}}\left(\widetilde{Q}_{l}^{n}+\frac{\widehat{\alpha}_{l} \pi_{l}^{n-1}-\widehat{\psi}_{l} \pi_{l}^{n-2}-P_{l}^{n-1}}{\widehat{\gamma}_{l}}\right) q\right|_{\Gamma_{l}}-\left(\boldsymbol{\nabla} \cdot \widetilde{\boldsymbol{u}}^{n}, q\right)_{\Omega_{\mathrm{f}}}
\end{aligned}
$$

for all $q \in R_{h}$.

3. End-of-step: Set $p^{n} \stackrel{\text { def }}{=} p^{n-1}+\delta p^{n}, \boldsymbol{u}^{n} \stackrel{\text { def }}{=} \widetilde{\boldsymbol{u}}^{n}-\frac{2 \tau}{3 \rho_{\mathrm{f}}} \nabla \delta p^{n} \in\left[L^{2}\left(\Omega_{\mathrm{f}}\right)\right]^{d}, P_{l}^{n}=\left.p^{n}\right|_{\Gamma_{l}}$ and compute $\left(Q_{l}^{n}, \pi_{l}^{n}\right) \in \mathbb{R}^{2}$ from the relations 25 .

Remark 3.8 It is well known that the stability of the pressure-incremental projection scheme requires that the finite element spaces for velocity and pressure satisfy an inf-sup condition (see [15] for an extended discussion).

\subsubsection{Extension to more complex lumped parameter models}

The algorithm and analysis presented above can be straightforwardly extended to more complex networks of lumped parameter models, made of resistances, capacitances, and inductances. These networks can be obtained, for instance, by connecting several Windkessel elements (see, e.g., [17, 19, 22] and the references therein). 
Let us consider the following general lumped parameter model

$$
\left\{\begin{array}{l}
C \frac{\mathrm{d} P}{\mathrm{~d} t}+R P=Q+H \Psi \\
L \frac{\mathrm{d} \Psi}{\mathrm{d} t}=-H^{\top} P
\end{array}\right.
$$

with

$$
P^{\mathrm{T}} \stackrel{\text { def }}{=}\left[\begin{array}{llllll}
P_{1} & \ldots & P_{n_{0 \mathrm{D}}} & \pi_{1} & \ldots & \pi_{n_{\pi}}
\end{array}\right]
$$

the collection of outlet $P_{l}, l=1, \ldots, n_{0 \mathrm{D}}$ and distal pressures $\pi_{j}, j=1, \ldots, n_{\pi}$, and $\Psi$ are the fluxes through the inductances, both representing the dynamical state of the lumped parameter model. Moreover, we assume that $Q(t) \in \mathbb{R}^{n_{p}}$ has the form

$$
Q^{\top} \stackrel{\text { def }}{=}\left[\begin{array}{llll}
Q_{1} & \ldots & Q_{n_{0 \mathrm{D}}} & 0 \ldots 0
\end{array}\right]
$$

with $Q_{l}$ the input flux at the outlet $\Gamma_{l}$.

In (36), $C, R, L$ denote symmetric capacitances, resistances and inductances matrices. This ensures the correct energy balance of the system in the 3D-0D time-space continuos formulation, namely

$$
\frac{\mathrm{d}}{\mathrm{d} t}\left(\frac{1}{2} P^{\boldsymbol{\top}} C P+\frac{1}{2} \Psi^{\boldsymbol{\top}} L \Psi\right)=-P^{\boldsymbol{\top}} R P+P^{\boldsymbol{\top}} Q
$$

since that the last term of the right-hand-side cancels out when coupling (36) with (1). As an example, the three-element Windkessel presented above, with an additional inductance $L_{p}$ parallel to $R_{p}$, can be rewritten in this format obtaining the following expressions for the system matrices

$$
C=\left[\begin{array}{cc}
0 & 0 \\
0 & C_{d}
\end{array}\right], \quad R=\left[\begin{array}{cc}
1 / R_{p} & -1 / R_{p} \\
-1 / R_{p} & 1 / R_{p}+1 / R_{d}
\end{array}\right], \quad H=\left[\begin{array}{c}
-1 \\
1
\end{array}\right], \quad L=L_{p} .
$$

More complex models can be represented in this format analagously.

Discretizing (36) in time (e.g., using a backward Euler scheme), we obtain

$$
P^{n}=A P^{n-1}+B Q^{n}+B H \Psi^{n-1},
$$

with

$$
B \stackrel{\text { def }}{=}(C / \tau+R)^{-1}, \quad A \stackrel{\text { def }}{=} B\left(C / \tau+\tau H L^{-1} H^{\top}\right) .
$$

In order to derive the 3D-0D implicit coupling scheme (Algorithm 2), we need explicit expressions for $Q_{l}^{n}, l=1, \ldots, n_{0 \mathrm{D}}$. These can be computed algebraically from (37) by first isolating the degrees-of-freedom on the outlets via

$$
\overline{\bar{B}}\left[\begin{array}{c}
Q_{1}^{n} \\
\vdots \\
Q_{n_{0 \mathrm{D}}}^{n}
\end{array}\right]=\left[\begin{array}{c}
P_{1}^{n} \\
\vdots \\
P_{n_{0 \mathrm{D}}}^{n}
\end{array}\right]-\bar{A} P^{n-1}-\bar{B} H \Psi^{n-1},
$$

with $\overline{()}$ denoting the first $n_{0 \mathrm{D}}$ rows and $\overline{\overline{(}}$ the first $n_{0 \mathrm{D}}$ rows and columns, respectively. Hence, we obtain the following expression

$\mathrm{RR} \mathrm{n}^{\circ} 7937$

$$
\left[\begin{array}{c}
Q_{1}^{n} \\
\vdots \\
Q_{n_{0 \mathrm{D}}}^{n}
\end{array}\right]=G\left[\begin{array}{c}
P_{1}^{n} \\
\vdots \\
P_{n_{0 \mathrm{D}}}^{n}
\end{array}\right]-G \bar{A} P^{n-1}-G \bar{B} H \Psi^{n-1}
$$


with $G=(\overline{\bar{B}})^{-1}$. Finally, by combining the latter with $(20)$, we obtain the following formulation of the pressure projection-Windkessel step (compare with (24)): Find $p^{n} \in R_{h}$ such that

$$
\begin{aligned}
\frac{\tau}{\rho_{\mathrm{f}}}\left(\boldsymbol{\nabla} p^{n}, \boldsymbol{\nabla} q\right)_{\Omega_{\mathrm{f}}}+\sum_{k, l=1}^{n_{0 \mathrm{D}}} g_{k, l}\left(\left.p^{n}\right|_{\Gamma_{k}}\right)\left(\left.q\right|_{\Gamma_{l}}\right) & \\
& =\sum_{l=1}^{n_{0 \mathrm{D}}}\left(\widetilde{Q}_{l}^{n}+\left.b_{l}^{n-1} q\right|_{\Gamma_{l}}\right)\left(\left.q\right|_{\Gamma_{l}}\right)-\left(\boldsymbol{\nabla} \cdot \widetilde{\boldsymbol{u}}^{n}, q\right)_{\Omega_{\mathrm{f}}}
\end{aligned}
$$

for all $q \in R_{h}$ and with $g_{k, l} \stackrel{\text { def }}{=}[G]_{k, l}, b_{l}^{n} \stackrel{\text { def }}{=}\left[G \bar{A} P^{n}+G \bar{B} H \Psi^{n}\right]_{l}$. Note that, in this general formulation, the pressure at the outlets can be coupled through the lumped parameter model since generally $g_{k, l} \neq 0$ for $k \neq l$.

Remark 3.9 Using the same arguments than in Section 3.2.1, the unconditional stability results of Theorem 3.7 can be extended to a fractional-step scheme with the generalized pressure projection formulation (38).

\section{Incompressible fluid-structure interaction}

Fractional-step time-marching schemes have been a valuable tool for the design of efficient solution methods for incompressible fluid-structure interaction (FSI) problems, yielding the projection semi-implicit coupling schemes [1, 9]. This coupling approach is based on the following three basic ideas:

- treat explicitly the geometrical non-linearities and the viscous-structure coupling, which reduces computational complexity;

- treat implicitly the presssure-structure coupling, which avoids numerical instability;

- perform this explicit-implicit splitting through a projection scheme in the fluid.

So far, the stability of this method has been analyzed within a simplified framework which enforces null pressure on the outlet boundaries (see [1, 9]). In this section the analysis is extended to the case of a lumped parameter modeling of the outlet boundaries, with a pressure-Poisson formulation of the projection step, based on the methods of Section 3

\subsection{Model problem}

For the sake of the analysis (see [9]), we consider as model problem a coupled linear system in which the fluid is described by the Stokes equations, in the fixed domain $\Omega_{\mathrm{f}}$, and the structure either by the classical linear elastodynamics equations or by equations based on linear thin-solid models (e.g., plate, shell, etc.). The reference domain of the solid is denoted by $\Omega_{\mathrm{s}}$. It will be either a domain or a 2 -manifold of $\mathbb{R}^{3}$ (in this later case the elastic domain is identified to its mid-surface). We denote by $\Sigma \stackrel{\text { def }}{=} \partial \Omega_{\mathrm{s}} \cap \partial \Omega_{\mathrm{f}}$ the fluid-structure interface. In the case the structure is described by thin-solid model we have $\overline{\Omega_{\mathrm{s}}}=\Sigma$ (see Figure 2). The resulting coupled system, describing the fluid velocity $\boldsymbol{u}: \Omega_{\mathrm{f}} \times \mathbb{R}^{+} \rightarrow \mathbb{R}^{3}$, fluid pressure $p: \Omega_{\mathrm{f}} \times \mathbb{R}^{+} \rightarrow \mathbb{R}$ and solid 


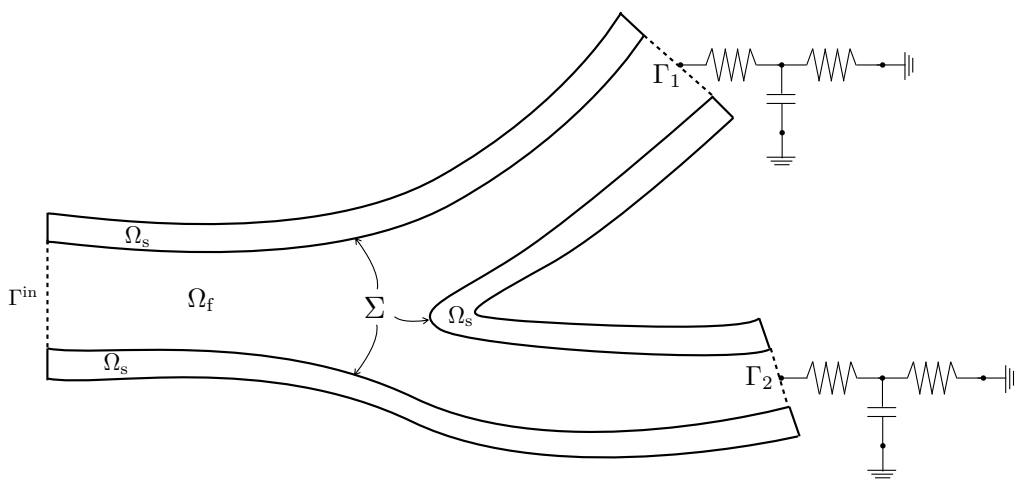

Figure 2: Examples of geometric configuration $\overline{\Omega_{\mathrm{s}}} \neq \Sigma\left(\overline{\Omega_{\mathrm{s}}}=\Sigma\right.$, see Figure 1).

displacement $\boldsymbol{y}: \Omega_{\mathrm{S}} \times \mathbb{R}^{+} \rightarrow \mathbb{R}^{3}$, is given by

$$
\begin{gathered}
\left\{\begin{aligned}
\rho_{\mathrm{f}} \partial_{t} \boldsymbol{u}-\boldsymbol{\nabla} \cdot \boldsymbol{\sigma}(\boldsymbol{u}, p)=\mathbf{0} & \text { in } \Omega_{\mathrm{f}}, \\
\boldsymbol{\nabla} \cdot \boldsymbol{u}=0 & \text { in } \Omega_{\mathrm{f}}, \\
\boldsymbol{u}=\boldsymbol{u}_{\mathrm{in}} & \text { on } \Gamma^{\text {in }}, \\
\boldsymbol{u}=\dot{\boldsymbol{y}} & \text { in } \Sigma,
\end{aligned}\right. \\
\left\{\begin{array}{l}
\rho_{\mathrm{s}}\left(\partial_{t} \dot{\boldsymbol{y}}, \boldsymbol{v}_{\mathrm{s}}\right)_{\Omega_{\mathrm{s}}}+a_{\mathrm{s}}\left(\boldsymbol{y}, \boldsymbol{v}_{\mathrm{s}}\right)=-\left(\boldsymbol{\sigma}(\boldsymbol{u}, p) \boldsymbol{n}_{\mathrm{f}}, \boldsymbol{v}_{\mathrm{s}}\right)_{\Sigma} \quad \forall \boldsymbol{v}_{s} \in \boldsymbol{W}, \\
\dot{\boldsymbol{y}}=\partial_{t} \boldsymbol{y} \text { in } \Omega_{\mathrm{s}},
\end{array}\right.
\end{gathered}
$$

completed with the lumped-parameter modeling (2)-(3) on the outlet boundary $\Gamma^{\text {out }}$ (see Figure 2). Here, $\rho_{\mathrm{s}}$ denotes the solid density, the abstract bilinear form $a_{\mathrm{s}}: \boldsymbol{W} \times \boldsymbol{W} \rightarrow \mathbb{R}$ describes the elastic behavior of the structure and $\boldsymbol{W}$ stands for its space of admissible displacements. It should be noted that the solid problem (40) has been written in weak form, which allows to treat in the same formulation the case of thin and thick solid models. The relations $\left({ }_{39}\right)_{4}$ and $(40)_{1}$ enforce the kinematic and kinetic interface coupling conditions, respectively. Note that the latter represents also the variational formulation of the structure subproblem. Though simplified, problem (39)-40 features some of the main numerical issues that appear in complex nonlinear fluid-structure interaction problems involving an incompressible fluid (see, e.g., [8]).

Let the quantity $E(t) \stackrel{\text { def }}{=} \frac{\rho_{\mathrm{f}}}{2}\|\boldsymbol{u}\|_{0, \Omega_{\mathrm{f}}}^{2}+\frac{\rho_{\mathrm{s}}}{2}\|\dot{\boldsymbol{y}}\|_{0, \Omega_{\mathrm{s}}}^{2}+\frac{1}{2}\|\boldsymbol{y}\|_{\mathrm{s}}^{2}+\sum_{l=1}^{n_{0 \mathrm{D}}} \frac{C_{\mathrm{d}, l}}{2} \pi_{l}^{2}$ denote the total (kinetic + potential) energy of the FSI-0D coupled system given by $(39)-(40)$ and $(2)-(3)$. Here, $\|\cdot\|_{\mathrm{s}}$ stands for the elastic energy norm of the solid, defined as $\|\boldsymbol{y}\|_{\mathrm{s}}^{2} \stackrel{\text { def }}{=} a_{\mathrm{s}}(\boldsymbol{y}, \boldsymbol{y})$. Assuming that $\boldsymbol{u}_{\text {in }}=\mathbf{0}$ (free system) and using a standard energy argument, we get the following identity

$$
E(t)+D(t)=E(0),
$$

with the dissipative term $D(t)>0$ given as in (4).

Note that in this model problem we do not consider the convective term in the fluid, hence we have to solve the fluid in a fixed domain in order to get the right energy balance across the fluid-structure interface $\Sigma$.

\subsubsection{Spatial discretization}

Let $\left\{\mathcal{T}_{\mathrm{s}, h}\right\}_{0<h \leq 1}$ be a quasi-uniform family of triangulations of the domain $\Omega_{\mathrm{s}}$. In order to ease the presentation, we assume that the fluid and solid triangulations $\mathcal{T}_{\mathrm{f}, h}$ and $\mathcal{T}_{\mathrm{s}, h}$ match at 
the interface $\Sigma$. For the discretization in space of the solid problem 40 , we consider continuous Lagrange finite element approximations, $\boldsymbol{W}_{h}$ of $\boldsymbol{W}$, which match the fluid velocity discretizations at the interface. Hence,

$$
\left\{\left.\boldsymbol{v}\right|_{\Sigma} \mid \boldsymbol{v} \in \boldsymbol{V}_{h}\right\}=\left\{\left.\boldsymbol{v}_{\mathrm{s}}\right|_{\Sigma} \mid \boldsymbol{v}_{\mathrm{s}} \in \boldsymbol{W}_{h}\right\}
$$

At last, we introduce the standard fluid-sided discrete lifting operator $\mathcal{L}_{h}: \boldsymbol{W}_{h} \rightarrow \boldsymbol{V}_{h}$, such that, the nodal values of $\mathcal{L}_{h} \boldsymbol{v}_{\mathrm{s}}$ vanish out of $\Sigma$ and $\left.\left(\mathcal{L}_{h} \boldsymbol{v}_{\mathrm{S}}\right)\right|_{\Sigma}=\left.\boldsymbol{v}_{\mathrm{s}}\right|_{\Sigma}$, for all $\boldsymbol{v}_{\mathrm{s}} \in \boldsymbol{W}_{h}$. In what follows we shall make use of the following continuity estimates (from [9, Lemma 1]) for the discrete lifting operator $\mathcal{L}_{h}$ :

$$
\left\|\mathcal{L}_{h} \boldsymbol{v}_{\mathrm{s}}\right\|_{0, \Omega_{\mathrm{f}}} \leq C_{\mathrm{L}} h^{\frac{1-\alpha}{2}}\left\|\boldsymbol{v}_{\mathrm{s}}\right\|_{0, \Omega_{\mathrm{s}}}, \quad\left\|\nabla \mathcal{L}_{h} \boldsymbol{v}_{\mathrm{s}}\right\|_{0, \Omega_{\mathrm{f}}} \leq C_{\mathrm{L}} h^{-\frac{1+\alpha}{2}}\left\|\boldsymbol{v}_{\mathrm{s}}\right\|_{0, \Omega_{\mathrm{s}}}
$$

for all $\boldsymbol{v}_{\mathrm{s}} \in \boldsymbol{W}_{h}$ and with the notation

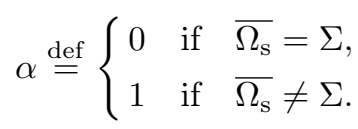

\subsubsection{Semi-implicit FSI scheme with implicit 3D-0D coupling}

In this case we consider numerical approximations of the coupled problem FSI-0D system (39)-(40) and (2)-(3) by combining the projection semi-implicit coupling scheme reported in [9] with the 3D-0D implicit coupling of Section 3.2. The resulting fully discrete time-marching procedure is reported in Algorithm 3. In the solid subproblem (47), the fluid residual terms are given by

$$
\begin{aligned}
&\left\langle\mathcal{R}_{\mu}\left(\widetilde{\boldsymbol{u}}^{n}\right), \boldsymbol{v}\right\rangle \stackrel{\text { def }}{=} \frac{\rho_{\mathrm{f}}}{\tau}\left(\widetilde{\boldsymbol{u}}^{n}, \boldsymbol{v}\right)_{\Omega_{\mathrm{f}}}+2 \mu\left(\boldsymbol{\epsilon}\left(\widetilde{\boldsymbol{u}}^{n}\right), \boldsymbol{\epsilon}(\boldsymbol{v})\right)_{\Omega_{\mathrm{f}}}-\frac{\rho_{\mathrm{f}}}{\tau}\left(\boldsymbol{u}^{n-1}, \boldsymbol{v}\right)_{\Omega_{\mathrm{f}}}, \\
&\left\langle\mathcal{R}_{p}\left(p^{n}, \boldsymbol{u}^{n}\right), \boldsymbol{v}\right\rangle \stackrel{\text { def }}{=} \frac{\rho_{\mathrm{f}}}{\tau}\left(\boldsymbol{u}^{n}, \boldsymbol{v}\right)_{\Omega_{\mathrm{f}}}-\frac{\rho_{\mathrm{f}}}{\tau}\left(\widetilde{\boldsymbol{u}}^{n}, \boldsymbol{v}\right)_{\Omega_{\mathrm{f}}}-\left(p^{n}, \boldsymbol{\nabla} \cdot \boldsymbol{v}\right)_{\Omega_{\mathrm{f}}} .
\end{aligned}
$$

Algorithm 3 (Semi-implicit FSI scheme with implicit 3D-0D coupling)

Let $\boldsymbol{u}^{0}=\boldsymbol{u}_{0}, \pi_{1}^{0}, \ldots, \pi_{n_{0 \mathrm{D}}}^{0} \in \mathbb{R}$ and $\boldsymbol{y}^{0}, \dot{\boldsymbol{y}}^{0} \in \boldsymbol{W}_{h}$ be given initial data. For $n \geq 1$ perform:

1. Viscous step: Find $\widetilde{\boldsymbol{u}}^{n} \in \boldsymbol{V}_{h}$ such that

$$
\left\{\begin{array}{l}
\left.\widetilde{\boldsymbol{u}}^{n}\right|_{\Sigma}=\left.\dot{\boldsymbol{y}}^{n-1}\right|_{\Sigma} \\
\left.\widetilde{\boldsymbol{u}}^{n}\right|_{\Gamma^{\mathrm{in}}}=\boldsymbol{u}_{\mathrm{in}}\left(t_{n}\right), \\
\frac{\rho_{\mathrm{f}}}{\tau}\left(\widetilde{\boldsymbol{u}}^{n}, \boldsymbol{v}\right)_{\Omega_{\mathrm{f}}}+2 \mu\left(\boldsymbol{\epsilon}\left(\widetilde{\boldsymbol{u}}^{n}\right), \boldsymbol{\epsilon}(\boldsymbol{v})\right)_{\Omega_{\mathrm{f}}}=\frac{\rho_{\mathrm{f}}}{\tau}\left(\boldsymbol{u}^{n-1}, \boldsymbol{v}\right)_{\Omega_{\mathrm{f}}}
\end{array}\right.
$$

for all $\boldsymbol{v} \in \boldsymbol{V}_{\Sigma \cup \Gamma^{\text {in }}, h}$. Thereafter set $\widetilde{Q}_{l}^{n} \stackrel{\text { def }}{=} \int_{\Gamma_{l}} \widetilde{\boldsymbol{u}}^{n} \cdot \boldsymbol{n}_{\mathrm{f}}$.

2. Implicit step (projection-Windkessel-solid step): Find $\left(p^{n}, \boldsymbol{u}^{n}, \boldsymbol{y}^{n}\right) \in R_{h} \times \boldsymbol{V}_{h} \times \boldsymbol{W}_{h}$ with 
$\dot{\boldsymbol{y}}^{n}=\partial_{\tau} \boldsymbol{y}^{n}$ and such that

$$
\begin{aligned}
& \left\{\begin{array}{l}
\frac{\tau}{\rho_{\mathrm{f}}}\left(\boldsymbol{\nabla} p^{n}, \boldsymbol{\nabla} q\right)_{\Omega_{\mathrm{f}}}+\sum_{l=1}^{n_{0 \mathrm{D}}} \frac{\left(\left.p^{n}\right|_{\Gamma_{l}}\right)\left(\left.q\right|_{\Gamma_{l}}\right)}{\gamma_{l}}=\left.\sum_{l=1}^{n_{0 \mathrm{D}}}\left(\widetilde{Q}_{l}^{n}+\frac{\alpha_{l} \pi_{l}^{n-1}}{\gamma_{l}}\right) q\right|_{\Gamma_{l}} \\
-\left(\boldsymbol{\nabla} \cdot \widetilde{\boldsymbol{u}}^{n}, q\right)_{\Omega_{\mathrm{f}}}-\left(\left(\dot{\boldsymbol{y}}^{n}-\widetilde{\boldsymbol{u}}^{n}\right) \cdot \boldsymbol{n}_{\mathrm{f}}, q\right)_{\Sigma},
\end{array}\right. \\
& \left\{\begin{array}{l}
\left.\boldsymbol{u}^{n}\right|_{\Gamma^{i n}}=\boldsymbol{u}_{\mathrm{in}}\left(t_{n}\right), \\
\left.\boldsymbol{u}^{n}\right|_{\Sigma}=\left.\dot{\boldsymbol{y}}^{n}\right|_{\Sigma}, \\
\frac{\rho_{\mathrm{f}}}{\tau}\left(\boldsymbol{u}^{n}, \boldsymbol{v}\right)_{\Omega_{\mathrm{f}}}=\frac{\rho_{\mathrm{f}}}{\tau}\left(\widetilde{\boldsymbol{u}}^{n}, \boldsymbol{v}\right)_{\Omega_{\mathrm{f}}}-\left(\boldsymbol{\nabla} p^{n}, \boldsymbol{v}\right)_{\Omega_{\mathrm{f}}},
\end{array}\right. \\
& \rho_{\mathrm{s}}\left(\partial_{\tau} \dot{\boldsymbol{y}}^{n}, \boldsymbol{v}_{\mathrm{s}}\right)_{\Omega_{\mathrm{s}}}+a_{\mathrm{s}}\left(\boldsymbol{y}^{n}, \boldsymbol{v}_{\mathrm{s}}\right)=-\left\langle\mathcal{R}_{\mu}\left(\widetilde{\boldsymbol{u}}^{n}\right), \mathcal{L}_{h} \boldsymbol{v}_{\mathrm{s}}\right\rangle-\left\langle\mathcal{R}_{p}\left(\boldsymbol{u}^{n}, p^{n}\right), \mathcal{L}_{h} \boldsymbol{v}_{\mathrm{s}}\right\rangle
\end{aligned}
$$

for all $\left(q, \boldsymbol{v}, \boldsymbol{v}_{\mathrm{s}}\right) \in R_{h} \times \boldsymbol{V}_{\Sigma \cup \Gamma^{\text {out }}, h} \times \boldsymbol{W}_{h}$.

Thereafter, set $P_{l}^{n}=\left.p^{n}\right|_{\Gamma_{l}}$ and compute $\left(Q_{l}^{n}, \pi_{l}^{n}\right) \in \mathbb{R}^{2}$ from the relations

$$
Q_{l}^{n}=\frac{P_{l}^{n}-\alpha_{l} \pi_{l}^{n-1}}{\gamma_{l}}, \quad \pi_{l}^{n}=\alpha_{l} \pi_{l}^{n-1}+\beta_{l} Q_{l}^{n}, \quad l=1, \ldots n_{0 \mathrm{D}}
$$

Let the quantities

$$
\begin{aligned}
& E^{n} \stackrel{\text { def }}{=} \frac{\rho_{\mathrm{f}}}{2}\left\|\boldsymbol{u}^{n}\right\|_{0, \Omega_{\mathrm{f}}}^{2}+\frac{\rho_{\mathrm{s}}}{2}\left\|\dot{\boldsymbol{y}}^{n}\right\|_{0, \Omega_{\mathrm{s}}}^{2}+\frac{1}{2}\left\|\boldsymbol{y}^{n}\right\|_{\mathrm{s}}^{2}+\sum_{l=1}^{n_{0 \mathrm{D}}} \frac{C_{\mathrm{d}, l}}{2}\left|\pi_{l}^{n}\right|^{2}, \\
& D^{n} \stackrel{\text { def }}{=} 2 \mu \sum_{m=1}^{n} \tau\left\|\boldsymbol{\epsilon}\left(\widetilde{\boldsymbol{u}}^{m}\right)\right\|_{0, \Omega_{\mathrm{f}}}^{2}+\sum_{m=1}^{n-1} \sum_{l=1}^{n_{0 \mathrm{D}}} \tau\left(\frac{\left|\pi_{l}^{m}\right|^{2}}{R_{\mathrm{d}, l}}+R_{\mathrm{p}, l}\left|Q_{l}^{m}\right|^{2}\right)
\end{aligned}
$$

for $n \geq 1$, denote the energy and physical dissipation of the discrete FSI-0D system. We then have the following energy based result.

Theorem 4.1 Let $\left\{\left(\widetilde{\boldsymbol{u}}^{n}, p^{n}, \boldsymbol{y}^{n}, \dot{\boldsymbol{y}}^{n}\right)\right\}_{n \geq 1}$ and $\left\{\left(Q_{l}^{n}, \pi_{l}^{n}, P_{l}^{n}\right)_{1 \leq l \leq n_{0 \mathrm{D}}}\right\}_{n \geq 1}$ be the approximated solution given by Algorithm 3, and assume that $\boldsymbol{u}_{\mathrm{in}}=\mathbf{0}$ (free system) and that the following condition holds

$$
\rho_{\mathrm{s}} \geq 3 C_{\mathrm{L}}\left(\rho_{\mathrm{f}} h^{1-\alpha}+\frac{\mu \tau}{h^{1+\alpha}}\right),
$$

with $\alpha$ given by (43). Then, following energy inequality holds

$$
E^{n}+D^{n} \lesssim E^{0}-\sum_{m=1}^{n-1} \frac{\tau^{2}}{\rho_{\mathrm{f}}}\left\|\Pi_{h}^{\perp}\left(\nabla p^{n}\right)\right\|_{0, \Omega_{\mathrm{f}}}^{2}
$$

with $\Pi_{h}: \boldsymbol{L}^{2}\left(\Omega^{\mathrm{f}}\right) \rightarrow \boldsymbol{V}_{\Sigma \cup \Gamma^{\mathrm{in}}, h}$ stands for the $L^{2}$-projection operator into $\boldsymbol{V}_{\Sigma \cup \Gamma^{\mathrm{in}}, h}$, and $\Pi_{h}^{\perp} \stackrel{\text { def }}{=}$ $I-\Pi_{h}$ for the corresponding orthogonal projection.

Proof. First, testing the viscous step (44) with $\boldsymbol{v}=\widetilde{\boldsymbol{u}}^{n}-\mathcal{L}_{h} \dot{\boldsymbol{y}}^{n-1}$ yields

$$
\begin{aligned}
\frac{\rho_{\mathrm{f}}}{2 \tau}\left(\left\|\widetilde{\boldsymbol{u}}^{n}\right\|_{0, \Omega_{\mathrm{f}}}^{2}-\left\|\boldsymbol{u}^{n-1}\right\|_{0, \Omega_{\mathrm{f}}}^{2}\right)+\frac{\rho_{\mathrm{f}}}{2 \tau}\left\|\widetilde{\boldsymbol{u}}^{n}-\boldsymbol{u}^{n-1}\right\|_{0, \Omega_{\mathrm{f}}}^{2} \\
+2 \mu\left\|\boldsymbol{\epsilon}\left(\widetilde{\boldsymbol{u}}^{n}\right)\right\|_{0, \Omega_{\mathrm{f}}}^{2}-\left\langle\mathcal{R}_{\mu}\left(\widetilde{\boldsymbol{u}}^{n}\right), \mathcal{L}_{h} \dot{\boldsymbol{y}}^{n-1}\right\rangle=0 .
\end{aligned}
$$


On the other hand, testing 46 with $\boldsymbol{v}=\boldsymbol{u}^{n}-\mathcal{L}_{h} \dot{\boldsymbol{y}}^{n}$ yields

$$
\begin{aligned}
\frac{\rho_{\mathrm{f}}}{2 \tau}\left(\left\|\boldsymbol{u}^{n}\right\|_{0, \Omega_{\mathrm{f}}}^{2}-\left\|\widetilde{\boldsymbol{u}}^{n}\right\|_{0, \Omega_{\mathrm{f}}}^{2}\right)+ & \frac{\rho_{\mathrm{f}}}{2 \tau}\left\|\boldsymbol{u}^{n}-\widetilde{\boldsymbol{u}}^{n}\right\|_{0, \Omega_{\mathrm{f}}}^{2} \\
& +\left(\boldsymbol{\nabla} p^{n}, \boldsymbol{u}^{n}\right)_{\Omega_{\mathrm{f}}}-\left(p^{n}, \dot{\boldsymbol{y}}^{n} \cdot \boldsymbol{n}_{\mathrm{f}}\right)_{\Sigma}-\left\langle\mathcal{R}_{p}\left(p^{n}, \boldsymbol{u}^{n}\right), \mathcal{L}_{h} \dot{\boldsymbol{y}}^{n}\right\rangle=0
\end{aligned}
$$

and taking $\boldsymbol{v}_{\mathrm{s}}=\dot{\boldsymbol{y}}^{n}$ in 47 yields

$$
\begin{aligned}
\frac{\rho_{\mathrm{s}}}{2} \partial_{\tau}\left\|\dot{\boldsymbol{y}}^{n}\right\|_{0, \Omega_{\mathrm{s}}}^{2}+\frac{\rho_{\mathrm{s}}}{2 \tau}\left\|\dot{\boldsymbol{y}}^{n}-\dot{\boldsymbol{y}}^{n-1}\right\|_{0, \Omega_{\mathrm{s}}}^{2}+\frac{1}{2} \partial_{\tau}\left\|\boldsymbol{y}^{n}\right\|_{\mathrm{s}}^{2}+\frac{1}{2 \tau}\left\|\boldsymbol{y}^{n}-\boldsymbol{y}^{n-1}\right\|_{\mathrm{s}}^{2} \\
=-\left\langle\mathcal{R}_{\mu}\left(\widetilde{\boldsymbol{u}}^{n}\right), \mathcal{L}_{h} \dot{\boldsymbol{y}}^{n}\right\rangle-\left\langle\mathcal{R}_{p}\left(p^{n}, \boldsymbol{u}^{n}\right), \mathcal{L}_{h} \dot{\boldsymbol{y}}^{n}\right\rangle .
\end{aligned}
$$

As a result, by adding the equalities $51-(53)$ we get

$$
\begin{aligned}
& \frac{\rho_{\mathrm{f}}}{2} \partial_{\tau}\left\|\boldsymbol{u}^{n}\right\|_{0, \Omega_{\mathrm{f}}}^{2}+2 \mu\left\|\boldsymbol{\epsilon}\left(\widetilde{\boldsymbol{u}}^{n}\right)\right\|_{0, \Omega_{\mathrm{f}}}^{2}+\frac{\rho_{\mathrm{s}}}{2} \partial_{\tau}\left\|\dot{\boldsymbol{y}}^{n}\right\|_{0, \Omega_{\mathrm{s}}}^{2}+\frac{1}{2} \partial_{\tau}\left\|\boldsymbol{y}^{n}\right\|_{\mathrm{s}}^{2} \\
& +\frac{\rho_{\mathrm{s}}}{2 \tau}\left\|\dot{\boldsymbol{y}}^{n}-\dot{\boldsymbol{y}}^{n-1}\right\|_{0, \Omega_{\mathrm{s}}}^{2}+\underbrace{\left(\boldsymbol{\nabla} p^{n}, \boldsymbol{u}^{n}\right)_{\Omega_{\mathrm{f}}}-\left(p^{n}, \dot{\boldsymbol{y}}^{n} \cdot \boldsymbol{n}_{\mathrm{f}}\right)_{\Sigma}}_{T_{1}}-\underbrace{\left\langle\mathcal{R}_{\mu}\left(\widetilde{\boldsymbol{u}}^{n}\right), \mathcal{L}\left(\dot{\boldsymbol{y}}^{n}-\dot{\boldsymbol{y}}^{n-1}\right)\right\rangle}_{T_{2}} \leq 0 .
\end{aligned}
$$

Following the argument used in [1, Appendix A], from (45) we infer that

$$
\widetilde{\boldsymbol{u}}^{n}=\boldsymbol{u}^{n}+\Pi_{h}^{\perp}\left(\widetilde{\boldsymbol{u}}^{n}-\mathcal{L}_{h} \dot{\boldsymbol{y}}^{n}\right)+\frac{\tau}{\rho_{\mathrm{f}}} \Pi_{h}\left(\boldsymbol{\nabla} p^{n}\right) .
$$

Thereafter, taking $q=p^{n}$ in 44 , integrating by parts in its right-hand side, and since $P_{l}^{n}=$ $\left.p^{n}\right|_{\Gamma_{l}}$, we have

$$
\frac{\tau}{\rho_{\mathrm{f}}}\left\|\boldsymbol{\nabla} p^{n}\right\|_{0, \Omega_{\mathrm{f}}}^{2}+\sum_{l=1}^{n_{0 \mathrm{D}}} \frac{P_{l}^{n}-\alpha_{l} \pi_{l}^{n-1}}{\gamma_{l}} P_{l}^{n}-\left(\widetilde{\boldsymbol{u}}^{n}, \boldsymbol{\nabla} p^{n}\right)_{\Omega_{\mathrm{f}}}+\left(p^{n}, \dot{\boldsymbol{y}}^{n} \cdot \boldsymbol{n}_{\mathrm{f}}\right)_{\Sigma}=0 .
$$

Now, by inserting (55) into this expression and using (31), we get

$$
\begin{aligned}
T_{1}= & \frac{\tau}{\rho_{\mathrm{f}}}\left\|\Pi_{h}^{\perp}\left(\nabla p^{n}\right)\right\|_{0, \Omega_{\mathrm{f}}}^{2}+\sum_{l=1}^{n_{0 \mathrm{D}}} \frac{P_{l}^{n}-\alpha_{l} \pi_{l}^{n-1}}{\gamma_{l}} P_{l}^{n}-\left(\Pi_{h}^{\perp}\left(\widetilde{\boldsymbol{u}}^{n}-\mathcal{L}_{h} \dot{\boldsymbol{y}}^{n}\right), \boldsymbol{\nabla} p^{n}\right)_{\Omega_{\mathrm{f}}} \\
\geq & \frac{\tau}{\rho_{\mathrm{f}}}\left\|\Pi_{h}^{\perp}\left(\boldsymbol{\nabla} p^{n}\right)\right\|_{0, \Omega_{\mathrm{f}}}^{2}+\frac{C_{\mathrm{d}, l}}{2} \partial_{\tau}\left|\pi_{l}^{n}\right|^{2}+\frac{1}{R_{\mathrm{d}, l}}\left|\pi_{l}^{n}\right|^{2}+R_{\mathrm{p}, l}\left|Q_{l}^{n}\right|^{2} \\
& -\underbrace{\left(\Pi_{h}^{\perp}\left(\widetilde{\boldsymbol{u}}^{n}-\mathcal{L}_{h} \dot{\boldsymbol{y}}^{n}\right), \nabla p^{n}\right)_{\Omega_{\mathrm{f}}}}_{T_{3}} .
\end{aligned}
$$

Therefore, by applying this lower bound to (54) we get

$$
\begin{array}{r}
\frac{\rho_{\mathrm{f}}}{2} \partial_{\tau}\left\|\boldsymbol{u}^{n}\right\|_{0, \Omega_{\mathrm{f}}}^{2}+\frac{\rho_{\mathrm{f}}}{2 \tau}\left\|\widetilde{\boldsymbol{u}}^{n}-\boldsymbol{u}^{n-1}\right\|_{0, \Omega_{\mathrm{f}}}^{2}+2 \mu\left\|\boldsymbol{\epsilon}\left(\widetilde{\boldsymbol{u}}^{n}\right)\right\|_{0, \Omega_{\mathrm{f}}}^{2}+\frac{\rho_{\mathrm{s}}}{2} \partial_{\tau}\left\|\dot{\boldsymbol{y}}^{n}\right\|_{0, \Omega_{\mathrm{s}}}^{2}+\frac{1}{2} \partial_{\tau}\left\|\boldsymbol{y}^{n}\right\|_{\mathrm{s}}^{2} \\
+\frac{\rho_{\mathrm{s}}}{2 \tau}\left\|\dot{\boldsymbol{y}}^{n}-\dot{\boldsymbol{y}}^{n-1}\right\|_{0, \Omega_{\mathrm{s}}}^{2}+\frac{\tau}{\rho_{\mathrm{f}}}\left\|\Pi_{h}^{\perp}\left(\boldsymbol{\nabla} p^{n}\right)\right\|_{0, \Omega_{\mathrm{f}}}^{2}+\frac{C_{\mathrm{d}, l}}{2} \partial_{\tau}\left|\pi_{l}^{n}\right|^{2}+\frac{1}{R_{\mathrm{d}, l}}\left|\pi_{l}^{n}\right|^{2}+R_{\mathrm{p}, l}\left|Q_{l}^{n}\right|^{2} \\
\leq T_{2}+T_{3} .
\end{array}
$$


Term $T_{2}$ can be bounded as in [9], using (42, which yields

$$
\begin{aligned}
T_{2} \leq & \frac{\rho_{\mathrm{f}}}{\tau}\left\|\widetilde{\boldsymbol{u}}^{n}-\boldsymbol{u}^{n-1}\right\|_{0, \Omega_{\mathrm{f}}}\left\|\mathcal{L}_{h}\left(\dot{\boldsymbol{y}}^{n}-\dot{\boldsymbol{y}}^{n-1}\right)\right\|_{0, \Omega_{\mathrm{f}}}+2 \mu\left\|\boldsymbol{\epsilon}\left(\widetilde{\boldsymbol{u}}^{n}\right)\right\|_{0, \Omega_{\mathrm{f}}}\left\|\boldsymbol{\epsilon}\left(\mathcal{L}_{h}\left(\dot{\boldsymbol{y}}^{n}-\dot{\boldsymbol{y}}^{n-1}\right)\right)\right\|_{0, \Omega_{\mathrm{f}}} \\
\leq & \varepsilon_{1} \frac{\rho_{\mathrm{f}}}{2 \tau}\left\|\widetilde{\boldsymbol{u}}^{n}-\boldsymbol{u}^{n-1}\right\|_{0, \Omega_{\mathrm{f}}}^{2}+\varepsilon_{2} \mu\left\|\boldsymbol{\epsilon}\left(\widetilde{\boldsymbol{u}}^{n}\right)\right\|_{0, \Omega_{\mathrm{f}}}^{2} \\
& +C_{\mathrm{L}}\left(\frac{\rho_{\mathrm{f}}}{2 \tau \varepsilon_{1}} h^{1-\alpha}+\frac{\mu}{\varepsilon_{2}} h^{-1-\alpha}\right)\left\|\dot{\boldsymbol{y}}^{n}-\dot{\boldsymbol{y}}^{n-1}\right\|_{0, \Omega_{\mathrm{s}}}^{2} .
\end{aligned}
$$

Term $T_{3}$ can be bounded following the argument used in [1], which yields

$$
\begin{aligned}
T_{3} & =\left(\Pi_{h}^{\perp}\left(\mathcal{L}_{h}\left(\dot{\boldsymbol{y}}^{n-1}-\dot{\boldsymbol{y}}^{n}\right)\right), \Pi_{h}^{\perp}\left(\nabla p^{n}\right)\right)_{\Omega_{\mathrm{f}}} \\
& \leq \varepsilon_{3} \frac{\tau}{2 \rho_{\mathrm{f}}}\left\|\Pi_{h}^{\perp}\left(\nabla p^{n}\right)\right\|_{0, \Omega_{\mathrm{f}}}^{2}+\frac{\rho_{\mathrm{f}}}{2 \tau \varepsilon_{3}} C_{\mathrm{L}} h^{1-\alpha}\left\|\dot{\boldsymbol{y}}^{n}-\dot{\boldsymbol{y}}^{n-1}\right\|_{0, \Omega_{\mathrm{s}}}^{2} .
\end{aligned}
$$

Hence, by inserting $(57)-58$ into 56 we get the energy estimate

$$
\begin{aligned}
\frac{\rho_{\mathrm{f}}}{2} \partial_{\tau}\left\|\boldsymbol{u}^{n}\right\|_{0, \Omega_{\mathrm{f}}}^{2}+\frac{\rho_{\mathrm{f}}}{2 \tau}\left(1-\varepsilon_{1}\right)\left\|\widetilde{\boldsymbol{u}}^{n}-\boldsymbol{u}^{n-1}\right\|_{0, \Omega_{\mathrm{f}}}^{2}+\mu\left(2-\varepsilon_{2}\right)\left\|\boldsymbol{\epsilon}\left(\widetilde{\boldsymbol{u}}^{n}\right)\right\|_{0, \Omega_{\mathrm{f}}}^{2}+\frac{\rho_{\mathrm{s}}}{2} \partial_{\tau}\left\|\dot{\boldsymbol{y}}^{n}\right\|_{0, \Omega_{\mathrm{s}}}^{2} \\
+\frac{1}{2} \partial_{\tau}\left\|\boldsymbol{y}^{n}\right\|_{\mathrm{s}}^{2}+\left[\frac{\rho_{\mathrm{s}}}{2 \tau}-C_{\mathrm{L}} \frac{\rho_{\mathrm{f}}}{2 \tau} h^{1-\alpha}\left(\frac{1}{\varepsilon_{1}}+\frac{1}{\varepsilon_{3}}\right)-C_{\mathrm{L}} \frac{\mu}{\varepsilon_{2}} h^{-1-\alpha}\right]\left\|\dot{\boldsymbol{y}}^{n}-\dot{\boldsymbol{y}}^{n-1}\right\|_{0, \Omega_{\mathrm{s}}}^{2} \\
\quad+\frac{\tau}{\rho_{\mathrm{f}}}\left(1-\frac{\varepsilon_{3}}{2}\right)\left\|\Pi_{h}^{\perp}\left(\nabla p^{n}\right)\right\|_{0, \Omega_{\mathrm{f}}}^{2}+\sum_{l=1}^{n_{0 \mathrm{D}}}\left(\frac{C_{\mathrm{d}, l}}{2} \partial_{\tau}\left|\pi_{l}^{n}\right|^{2}+\frac{1}{R_{\mathrm{d}, l}}\left|\pi_{l}^{n}\right|^{2}+R_{\mathrm{p}, l}\left|Q_{l}^{n}\right|^{2}\right) \leq 0 .
\end{aligned}
$$

At last, the energy estimate f50 follows by taking in the latter $\varepsilon_{1}=\frac{1}{2}, \varepsilon_{2}=\varepsilon_{3}=1$, summing over $n$ and using $\sqrt{49}$, which completes the proof.

Proposition 4.1 guarantees the conditional stability of Algorithm 3. Note that the stability condition is similar to the one obtained in [9] with a Darcy-like formulation of the projection step. The estimate (50) corresponds to the discrete counterpart of (41), the right-hand side of (50) is a dissipative numerical term related to the natural pressure stabilization of the projection scheme. Moreover, extensive numerical simulations with the semi-implicit FSI coupling scheme in physiological regimes (see [9, 8, 20]) suggest that condition 449 is not necessary for stability.

\subsubsection{Semi-implicit FSI scheme with explicit 3D-0D coupling}

We now consider numerical approximations of the coupled problem FSI-0D system (39)- 40, and (2)-(3) by combining the projection semi-implicit coupling scheme reported in [9] with the $3 \mathrm{D}-0 \mathrm{D}$ explicit coupling of Section 3.1. The resulting fully discrete time-marching procedure is reported in Algorithm 4.

\section{Algorithm 4 (Semi-implicit FSI scheme with explicit 3D-0D coupling)}

Let $\boldsymbol{u}^{0}=\boldsymbol{u}_{0}, \pi_{1}^{0}, \ldots, \pi_{n_{0 \mathrm{D}}}^{0} \in \mathbb{R}$ and $\boldsymbol{y}^{0}, \dot{\boldsymbol{y}}^{0} \in \boldsymbol{W}_{h}$ be given initial data. For $n \geq 1$ perform:

1. Viscous step: Find $\widetilde{\boldsymbol{u}}^{n} \in \boldsymbol{V}_{h}$ such that

$$
\left\{\begin{array}{l}
\left.\widetilde{\boldsymbol{u}}^{n}\right|_{\Sigma}=\left.\dot{\boldsymbol{y}}^{n-1}\right|_{\Sigma}, \\
\left.\widetilde{\boldsymbol{u}}^{n}\right|_{\Gamma^{\text {in }}}=\boldsymbol{u}_{\mathrm{in}}\left(t_{n}\right), \\
\frac{\rho_{\mathrm{f}}}{\tau}\left(\widetilde{\boldsymbol{u}}^{n}, \boldsymbol{v}\right)_{\Omega_{\mathrm{f}}}+2 \mu\left(\boldsymbol{\epsilon}\left(\widetilde{\boldsymbol{u}}^{n}\right), \boldsymbol{\epsilon}(\boldsymbol{v})\right)_{\Omega_{\mathrm{f}}}=\frac{\rho_{\mathrm{f}}}{\tau}\left(\boldsymbol{u}^{n-1}, \boldsymbol{v}\right)_{\Omega_{\mathrm{f}}}
\end{array}\right.
$$

for all $\boldsymbol{v} \in \boldsymbol{V}_{\Sigma \cup \Gamma^{\text {in }}, h}$. Thereafter set $\widetilde{Q}_{l}^{n} \stackrel{\text { def }}{=} \int_{\Gamma_{l}} \widetilde{\boldsymbol{u}}^{n} \cdot \boldsymbol{n}_{\mathrm{f}}$. 
2. Windkessel step: For $l=1, \ldots, n_{0 \mathrm{D}}$, compute $\left(Q_{l}^{n}, \pi_{l}^{n}, P_{l}^{n}\right) \in \mathbb{R}^{3}$ from

$$
\left\{\begin{array}{l}
Q_{l}^{n}=\widetilde{Q}_{l}^{n}, \\
C_{\mathrm{d}, l} \partial_{\tau} \pi_{l}^{n}+\frac{\pi_{l}^{n}}{R_{\mathrm{d}, l}}=Q_{l}^{n}, \\
P_{l}^{n}=R_{\mathrm{p}, l} Q_{l}^{n}+\pi_{l}^{n} .
\end{array}\right.
$$

3. Implicit projection-solid step: Find $\left(p^{n}, \boldsymbol{u}^{n}, \boldsymbol{y}^{n}\right) \in R_{h} \times \boldsymbol{V}_{h} \times \boldsymbol{W}_{h}$ with $\dot{\boldsymbol{y}}^{n}=\partial_{\tau} \boldsymbol{y}^{n}$ and such that

$$
\begin{aligned}
& \left\{\begin{array}{l}
\left.p^{n}\right|_{\Gamma_{l}}=P_{l}^{n}, \quad l=1, \ldots, n_{0 \mathrm{D}}, \\
\frac{\tau}{\rho_{\mathrm{f}}}\left(\boldsymbol{\nabla} p^{n}, \boldsymbol{\nabla} q\right)_{\Omega_{\mathrm{f}}}=-\left(\boldsymbol{\nabla} \cdot \widetilde{\boldsymbol{u}}^{n}, q\right)_{\Omega_{\mathrm{f}}}-\left(\left(\dot{\boldsymbol{y}}^{n}-\widetilde{\boldsymbol{u}}^{n}\right) \cdot \boldsymbol{n}_{\mathrm{f}}, q\right)_{\Sigma}
\end{array}\right. \\
& \left\{\begin{array}{l}
\left.\boldsymbol{u}^{n}\right|_{\Gamma_{\mathrm{in}}}=\boldsymbol{u}_{\mathrm{in}}\left(t_{n}\right), \\
\left.\boldsymbol{u}^{n}\right|_{\Sigma}=\left.\dot{\boldsymbol{y}}^{n}\right|_{\Sigma}, \\
\frac{\rho_{\mathrm{f}}}{\tau}\left(\boldsymbol{u}^{n}, \boldsymbol{v}\right)_{\Omega_{\mathrm{f}}}=\frac{\rho_{\mathrm{f}}}{\tau}\left(\widetilde{\boldsymbol{u}}^{n}, \boldsymbol{v}\right)_{\Omega_{\mathrm{f}}}-\left(\boldsymbol{\nabla} p^{n}, \boldsymbol{v}\right)_{\Omega_{\mathrm{f}}},
\end{array}\right. \\
& \rho_{\mathrm{s}}\left(\partial_{\tau} \dot{\boldsymbol{y}}^{n}, \boldsymbol{v}_{\mathrm{s}}\right)_{\Omega_{\mathrm{s}}}+a_{\mathrm{s}}\left(\boldsymbol{y}^{n}, \boldsymbol{v}_{\mathrm{s}}\right)=-\left\langle\mathcal{R}_{\mu}\left(\widetilde{\boldsymbol{u}}^{n}\right), \mathcal{L}_{h} \boldsymbol{v}_{\mathrm{s}}\right\rangle-\left\langle\mathcal{R}_{p}\left(\boldsymbol{u}^{n}, p^{n}\right), \mathcal{L}_{h} \boldsymbol{v}_{\mathrm{s}}\right\rangle
\end{aligned}
$$

for all $\left(q, \boldsymbol{v}, \boldsymbol{v}_{\mathrm{s}}\right) \in R_{\Gamma^{\mathrm{out}}, h} \times \boldsymbol{V}_{\Sigma \cup \Gamma^{\mathrm{in}}, h} \times \boldsymbol{W}_{h}$.

The following proposition provides an energy estimate for the approximations provided by Algorithm 4.

Theorem 4.2 Let $\left\{\left(\widetilde{\boldsymbol{u}}^{n}, p^{n}, \boldsymbol{y}^{n}, \dot{\boldsymbol{y}}^{n}\right)\right\}_{n>1}$ and $\left\{\left(Q_{l}^{n}, \pi_{l}^{n}, P_{l}^{n}\right)_{1 \leq l \leq n_{0 \mathrm{D}}}\right\}_{n>1}$ be the approximated solution given by Algorithm 4 and assume that $\boldsymbol{u}_{\mathrm{in}}=\mathbf{0}$ (free system). Then, under the condition (49), the following energy inequality holds

$$
\begin{aligned}
E^{n}+D^{n} \lesssim E^{0}-\sum_{m=1}^{n-1} \frac{\tau^{2}}{2 \rho_{\mathrm{f}}}\left\|\Pi_{h}^{\perp}\left(\nabla p^{m}\right)\right\|_{0, \Omega_{\mathrm{f}}}^{2}+\sum_{m=1}^{n-1} \tau\left(\boldsymbol{\nabla} \cdot \widetilde{\boldsymbol{u}}^{m}, \phi^{m}\right)_{\Omega_{\mathrm{f}}} \\
+\sum_{m=1}^{n-1} \frac{\tau^{2}}{\rho_{\mathrm{f}}}\left(\boldsymbol{\nabla} p^{m}, \boldsymbol{\nabla} \phi^{m}\right)_{\Omega_{\mathrm{f}}}+\sum_{m=1}^{n-1} \tau\left(\dot{\boldsymbol{y}}^{m} \cdot \boldsymbol{n}_{\mathrm{f}}, \phi^{m}\right)_{\Sigma}
\end{aligned}
$$

Proof. The result follows by combining the arguments involved in the proofs of Propositions 3.2 and 4.1 . Hence, only partial details are given. The main difference lies on the estimation of term $T_{1}$ in (54). Since $q \in R_{\Gamma^{\text {out }}, h}$, integration by parts in 61) gives

$$
\left(\nabla p^{n}, \nabla q\right)_{\Omega_{\mathrm{f}}}=\frac{\rho_{\mathrm{f}}}{\tau}\left(\widetilde{\boldsymbol{u}}^{n}, \nabla q\right)_{\Omega_{\mathrm{f}}}-\left(\dot{\boldsymbol{y}}^{n} \cdot \boldsymbol{n}_{\mathrm{f}}, q\right)_{\Sigma},
$$

so that by testing with $q=\left(p^{n}-\phi^{n}\right) \in R_{\Gamma^{\text {out }}, h}$ we get

$$
\frac{\tau}{\rho_{\mathrm{f}}}\left\|\boldsymbol{\nabla} p^{n}\right\|_{0, \Omega_{\mathrm{f}}}^{2}-\left(\widetilde{\boldsymbol{u}}^{n}, \boldsymbol{\nabla} p^{n}\right)_{\Omega_{\mathrm{f}}}+\left(\dot{\boldsymbol{y}}^{n} \cdot \boldsymbol{n}_{\mathrm{f}}, p^{n}\right)_{\Sigma}+\left(\widetilde{\boldsymbol{u}}^{n}-\frac{\tau}{\rho_{\mathrm{f}}} \boldsymbol{\nabla} p^{n}, \boldsymbol{\nabla} \phi^{n}\right)_{\Omega_{\mathrm{f}}}-\left(\dot{\boldsymbol{y}}^{n} \cdot \boldsymbol{n}_{\mathrm{f}}, \phi^{n}\right)_{\Sigma}=0
$$


As in the proof of Proposition 4.1, from $(62$ we get 55 . Hence, inserting this expression into (65) we get

$$
\begin{aligned}
-\left(\nabla p^{n}, \boldsymbol{u}^{n}\right)_{\Omega_{\mathrm{f}}}+\left(p^{n}, \dot{\boldsymbol{y}}^{n} \cdot \boldsymbol{n}_{\mathrm{f}}\right)_{\Sigma}+\frac{\tau}{\rho_{\mathrm{f}}}\left\|\Pi_{h}^{\perp}\left(\nabla p^{n}\right)\right\|_{0, \Omega_{\mathrm{f}}}^{2}-\left(\Pi_{h}^{\perp}\left(\widetilde{\boldsymbol{u}}^{n}-\mathcal{L}_{h} \dot{\boldsymbol{y}}^{n}\right), \boldsymbol{\nabla} p^{n}\right)_{\Omega_{\mathrm{f}}} \\
=-\left(\widetilde{\boldsymbol{u}}^{n}-\frac{\tau}{\rho_{\mathrm{f}}} \boldsymbol{\nabla} p^{n}, \boldsymbol{\nabla} \phi^{n}\right)_{\Omega_{\mathrm{f}}}+\left(\dot{\boldsymbol{y}}^{n} \cdot \boldsymbol{n}_{\mathrm{f}}, \phi^{n}\right)_{\Sigma} \\
-\sum_{l=1}^{n_{0 \mathrm{D}}} Q_{l}^{n} P_{l}^{n}+\left(\boldsymbol{\nabla} \cdot \widetilde{\boldsymbol{u}}^{n}, \phi^{n}\right)_{\Omega_{\mathrm{f}}}+\frac{\tau}{\rho_{\mathrm{f}}}\left(\boldsymbol{\nabla} p^{n}, \boldsymbol{\nabla} \phi^{n}\right)_{\Omega_{\mathrm{f}}}+\left(\dot{\boldsymbol{y}}^{n} \cdot \boldsymbol{n}_{\mathrm{f}}, \phi^{n}\right)_{\Sigma}
\end{aligned}
$$

and the estimate 64 follows using the same arguments than in the proof of Proposition 4.1

Remark 4.3 A comparison of the energy estimates (16) and (64) suggests that the fluid-solid interaction introduces and additional destabilizing effect in the explicit splitting of the 3D-OD coupling 12 , due to the presence of the artificial interface term

$$
\sum_{m=1}^{n-1} \tau\left(\dot{\boldsymbol{y}}^{m} \cdot \boldsymbol{n}_{\mathrm{f}}, \phi^{m}\right)_{\Sigma} .
$$

In particular, it is worth noting that the observation made in Remark 3.4 for the case of a single outlet is not valid in the FSI framework, since the above term does not vanish for $\phi^{m}=P^{m}$ in $\Omega_{\mathrm{f}}$. This point will be illustrated trough numerical experiments in Section 5.2.

\section{$5 \quad$ Numerical experiments}

In this section we present two numerical experiments that confirm the analysis of the previous sections.

\subsection{Blood flow in a patient-specific aorta}

Our first example is a pure Navier-Stokes flow within a patient-specific aorta with repaired coarctation (see Figures 3-Left-Center). The geometry comes from the euHeart database ${ }^{1}$. A

\begin{tabular}{l|c|c|c|c} 
& Outlet 1 & Outlet 2 & Outlet 3 & Outlet 4 \\
\hline$R_{p}\left(\mathrm{dyn} \cdot \mathrm{s} \cdot \mathrm{cm}^{-5}\right)$ & 250 & 683 & 615 & 94 \\
$R_{d}\left(\mathrm{dyn} \cdot \mathrm{s} \cdot \mathrm{cm}^{-5}\right)$ & $10^{4}$ & $1.296 \cdot 10^{4}$ & $1.1664 \cdot 10^{4}$ & $0.1794 \cdot 10^{4}$ \\
$C\left(\mathrm{~cm}^{5} \cdot \mathrm{dyn}^{-1}\right)$ & $4 \cdot 10^{-4}$ & $2 \cdot 10^{-4}$ & $2 \cdot 10^{-4}$ & $14 \cdot 10^{-4}$
\end{tabular}

Table 1: Parameters for the Windkessel's model. The outlets are ordered in direction of the flow.

segment growing registration algorithm (see 2, 3]) was used for the segmentation of the geometry from the medical image. The resulting surface was pre-processed with 3-matic (Materialise) and the final mesh was generated with Gmsh [13. The inflow curve used as boundary condition (Figure 4-Right, black-line) was obtained from the same patient with Phase Contrast MRI. The initial constant pressure was set to $47 \mathrm{mmHg}$, and the Windkessel parameters (reported

$1_{\text {www. euheart.eu }}$ 

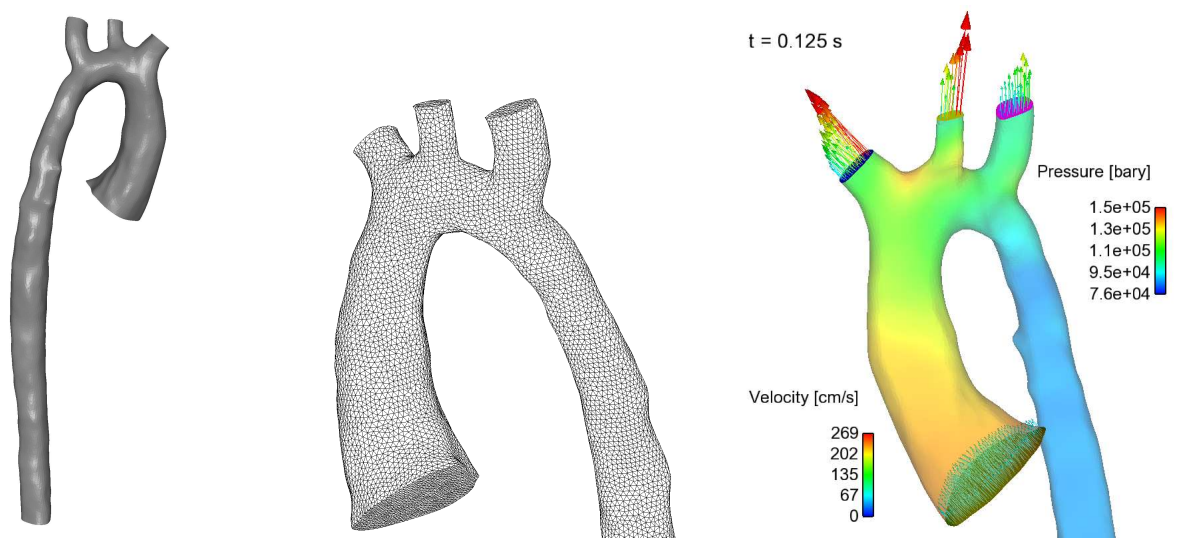

Figure 3: Patient-specific aorta. Left: geometry. Center: surface mesh. Right: simulation results for the aorta (vector velocity field and pressure distribution).
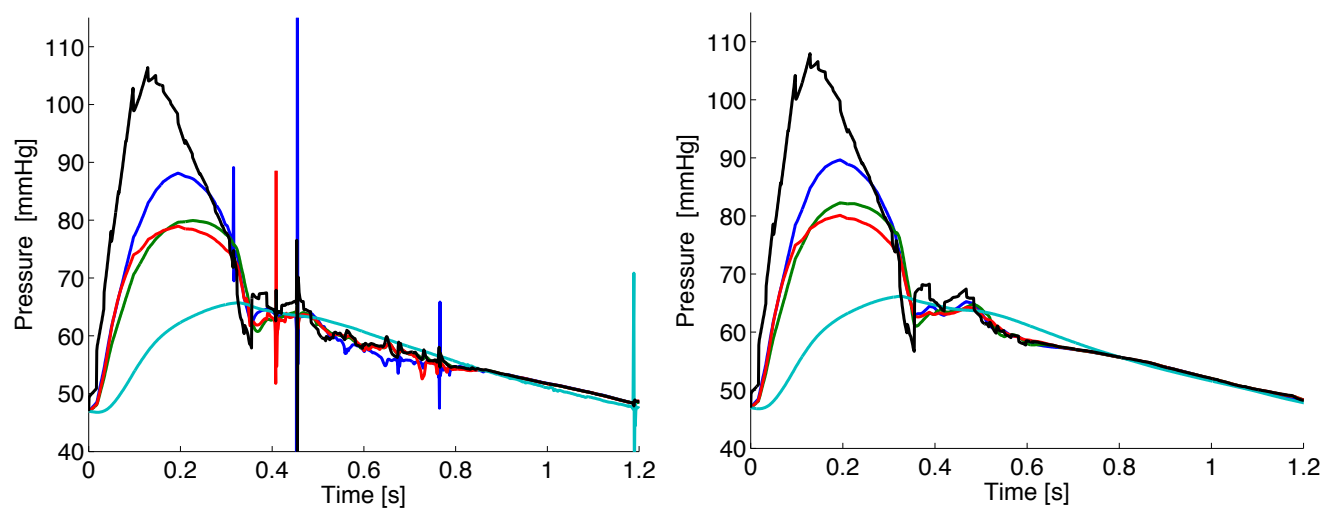

Figure 4: Mean pressures at inlet (black) and outlets 1 to 4 (blue, green, red, cyan). Explicit (left) and implicit (right) schemes.

in Table 11 where calibrated in order to approximate the measured pressure at the coarctation and the measured flow on each outlet. The physical parameters of the fluid are $\mu=0.035$ Po and $\rho_{\mathrm{f}}=1 \mathrm{gr} / \mathrm{cm}^{3}$. For the numerical simulation, we use $\mathbb{P}_{1}$ finite elements for both pressure and velocity fields (SUPG stabilization in the viscous-convective step, where we assume that the first perform the Chorin-Temam splitting and then we stabilize) and a time step $\tau=10^{-3} \mathrm{~s}$. A snapshot of the simulation results is shown in Figure 3.Right. Figures 4 and 5 show the pressure and flow results for both schemes (Algorithms 1 and 2, respectively). The spurious oscillations in the approximation provided by the explicit 3D-0D coupling scheme are clearly visible for all the outlet pressures and (less pronounced) for some outlet fluxes, while the implicit formulation guarantees stability within the whole cardiac cycle. Hence, these results are in agreement with the stability estimates provided by Theorems 3.2 and 3.7. As further validation of the numerical scheme, Figure 6 shows the pressures and flows curves computed using a monolithic solver, implicitely coupled with the 0D-model, showing a satisfactory agreement of the results. 

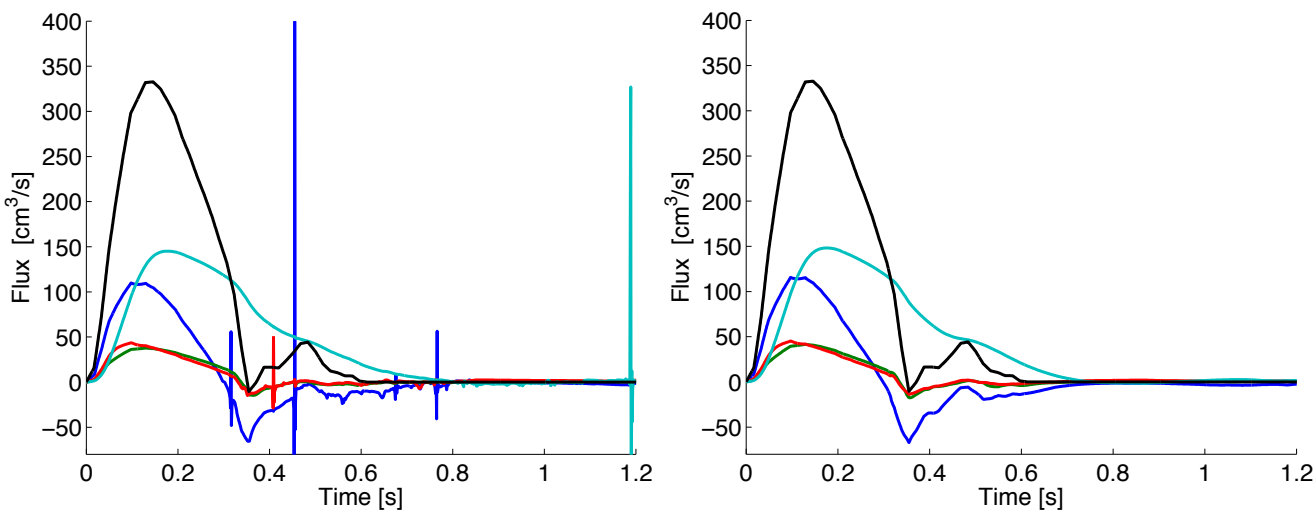

Figure 5: Fluxes for inlet (black) and outlets 1 to 4 (blue, green, red, cyan). Explicit (left) and implicit (right) schemes.
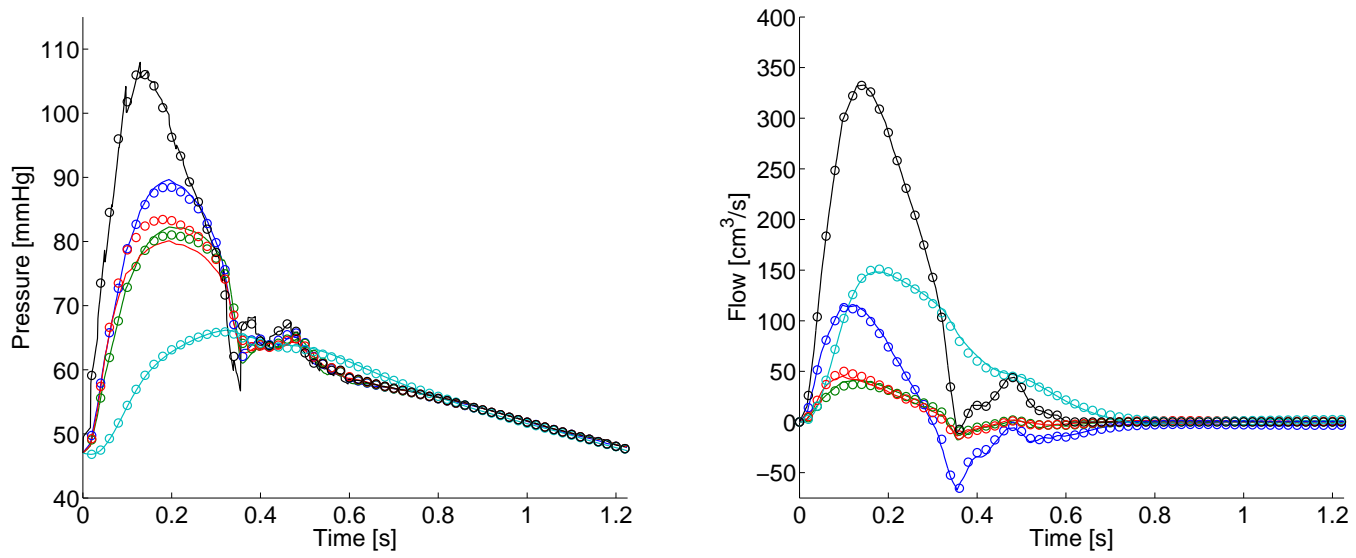

Figure 6: Pressures (left) and fluxes (right) for inlet (black) and outlets 1-4 (blue, green, red, cyan) obtained with a monolithic method (circles) and with the projection method (continuos) using an implicit 3D-0D coupling.

\subsection{Fluid-structure interaction in an idealized AAA}

We consider an idealized abdominal aortic aneurysm (AAA) of length $22.95 \mathrm{~cm}$, minimal diameter $1.7 \mathrm{~cm}$ (tubular part), maximal diameter $4.98 \mathrm{~cm}$ (aneurysm) and wall thickness $0.2 \mathrm{~cm}$ (see Figure 7 (left)). The structure is described by the linear elastodynamics equations and the fluid by the incompressible Navier-Stokes equations in a moving domain (ALE formalism). We considered the non-linear counterpart of Algorithms 3 and 4 using the projection semi-implicit coupling scheme reported in 9]. The solid has Young's modulus $1 M P a$, Poisson ratio is 0.46 and density $1.2 \mathrm{~g} / \mathrm{cm}^{3}$. The fluid viscosity is $\mu=0.035 P_{o}$ and its density $1 \mathrm{~g} / \mathrm{cm}^{3}$. The inlet and the outlet parts of the solid are clamped. In the fluid, a parabolic velocity profile is enforced at the inlet, with a realistic inflow (peak velocity $\approx 96 \mathrm{~cm} / \mathrm{s}$ ). The Windkessel parameters are $R_{p}=700 \mathrm{dyn} \cdot \mathrm{s} \cdot \mathrm{cm}^{-5}, R_{d}=5 \cdot 10^{3} \mathrm{dyn} \cdot \mathrm{s} \cdot \mathrm{cm}^{-5}$ and $C=2 \cdot 10^{-4} \mathrm{~cm}^{5} \cdot \mathrm{dyn}^{-1}$. At $t=0$, 

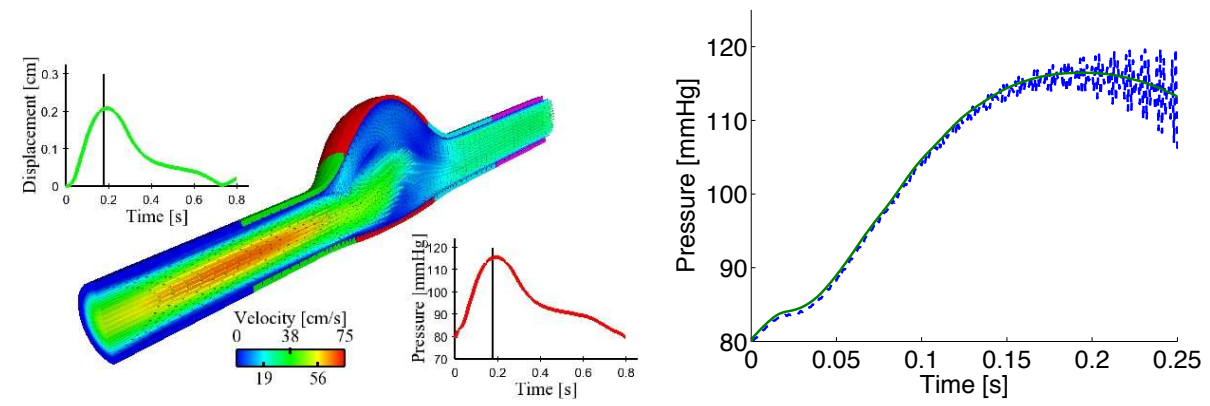

Figure 7: Left: snapshot of the velocity field (left) inside the idealized AAA, the curves show outlet pressure and displacements of the aneurysm wall. Right: Windkessel pressure for the AAA, comparison between implicit (solid line) and explicit (dashed line) fluid-Windkessel coupling.

the pressure is constant and equal to $80 \mathrm{mmHg}$, whereas all the other state variables are zero. During the whole simulation, the stress received by the structure is corrected by the initial one, and the solid only responds to the difference with the diastolic phase. In this way, the load applied to the structure is kept reasonable small so that the linearity assumptions holds.

The results are summarized in Figure 7 (right), showing the Windkessel pressures in time for a time step $P$ for $\tau=0.001 s$. Note that the semi-implicit algorithm with explicit 0D-3D coupling (Algorithm 4) is unstable whereas with the implicit 3D-0D treatment (Algorithm 3) the numerical solution remains stable. In fact, from the results one can infer that the interface term outlined in Remark 4.3, namely $\left(\dot{\boldsymbol{y}}^{n}, P^{n} \boldsymbol{n}_{\mathrm{f}}\right)_{\Sigma}$, injects a positive artificial power into the system (an increased pressure $P^{n}>0$ leads to $\dot{\boldsymbol{y}}^{n} \cdot \boldsymbol{n}_{\mathrm{f}}>0$ ).

\section{Conclusions}

In this paper we formulated and analyzed the treatment of a 3D-0D coupling between the 3D distributed (fluid, fluid-structure interaction) models and a set of Windkessel 0D models. The key ingredient in the proposed schemes is the use of a fractional-step time-marching in the 3D compartment. For purely fluid problems with multiple outlets, our energy based stability analysis showed that numerical instabilities might appear when using an explicit 3D-0D coupling. Interestingly, this result also holds with a single outlet in the case of fluid-structure interaction. We proposed to overcome this issues via an implicit treatment of the 3D-0D coupling, which involves a negligible additional computational cost with respect to the explicit strategy. These theoretical expectations were confirmed by numerical experiments in realistic geometries and physiological data.

\section{Acknowledgements}

The authors are thankful to Jean-Frédéric Gerbeau and Romain Guibert (INRIA) for valuable discussions, Philipp Beerbaum and Israel Valverde (King's College London), for the acquisition of the patient data, and David Barber, Rod Hose and Cristina Staicu (University of Sheffield) for the segmented geometry. This work was partially founded by the Seventh Framework Program (FP7/2007-2013) of the European Community, under grant agreement 224495 (euHeart project). 


\section{References}

[1] M. Astorino, F. Chouly, and M. A. Fernández. Robin based semi-implicit coupling in fluidstructure interaction: Stability analysis and numerics. SIAM J. Sci. Comp., 31(6):40414065, 2009

[2] D.C. Barber, E. Oubel, A.F. Frangi, and D.R. Hose. Efficient computational fluid dynamics mesh generation by image registration. Med. Image Anal., 11(6):648-662, 2007.

[3] D.C. Barber, C. Staicu, I. Valverde, P. Beerbaum, and D.R. Hose. Registration based segment growing for vascular segmentation. Submitted to IEEE T Med Imaging, 2012.

[4] Y. Bazilevs, J.R. Gohean, T.J.R. Hughes, R.D. Moser, and Y. Zhang. Patient-specific isogeometric fluid-structure interaction analysis of thoracic aortic blood flow due to implantation of the Jarvik 2000 left ventricular assist device. Comput. Methods Appl. Mech. Engrg., 198(45-46):3534-3550, 2009.

[5] C. Bertoglio. Forward and inverse problems in fluid-structure interaction. Application to hemodynamics. PhD thesis, Université Pierre et Marie Curie - Paris VI, 2012. http: //tel.archives-ouvertes.fr/tel-00768188

[6] A. J. Chorin. Numerical solution of the Navier-Stokes equations. Math. Comp., 22:745-762, 1968.

[7] A. Ern and J.-L. Guermond. Theory and practice of finite elements, volume 159 of Applied Mathematical Sciences. Springer-Verlag, New York, 2004.

[8] M.A. Fernández. Coupling schemes for incompressible fluid-structure interaction: implicit, semi-implicit and explicit. S $\overrightarrow{\mathrm{e}} M A$ J., 55:59-108, 2011.

[9] M.A. Fernández, J.F. Gerbeau, and C. Grandmont. A projection semi-implicit scheme for the coupling of an elastic structure with an incompressible fluid. Int. J. Num. Meth. Engrg., $69(4): 794-821,2007$.

[10] L. Formaggia, A. Moura, and F. Nobile. On the stability of the coupling of 3D and 1D fluid-structure interaction models for blood flow simulations. M2AN Math. Model. Numer. Anal., 41(4):743-769, 2007.

[11] L. Formaggia, A. Quarteroni, and A. Veneziani, editors. Cardiovascular Mathematics. Modeling and simulation of the circulatory system, volume 1 of Modeling, Simulation and Applications. Springer, 2009.

[12] O. Frank. Die Grundform des arteriellen Pulses. Zeitschrift für Biologie, 37:483-526, 1899.

[13] C. Geuzaine and J.-F. Remacle. Gmsh: A 3-D finite element mesh generator with built-in pre- and post-processing facilities. Int. J. Num. Meth. Engnr., 79(11):1309-1331, 2009.

[14] J.-L. Guermond, P. Minev, and J. Shen. Error analysis of pressure-correction schemes for the time-dependent Stokes equations with open boundary conditions. SIAM J. Numer. Anal., 43(1):239-258, 2005.

[15] J.-L. Guermond, P. Minev, and Jie Shen. An overview of projection methods for incompressible flows. Comput. Methods Appl. Mech. Engrg., 195(44-47), 2006.

$\mathrm{RR} \mathrm{n}^{\circ} 7937$ 
[16] J.-L. Guermond and L. Quartapelle. On stability and convergence of projection methods based on pressure Poisson equation. Internat. J. Numer. Methods Fluids, 26(9):1039-1053, 1998.

[17] T. Korakianitis and Y. Shi. A concentrated parameter model for the human cardiovascular system including heart valve dynamics and atrioventricular interaction. Medical Engineering E Physics, 28:613-628, 2006.

[18] A.C.I. Malossi, P.J. Blanco, S. Deparis, and A. Quarteroni. Algorithms for the partitioned solution of weakly coupled fluid models for cardiovascular flows. Int. J. Numer. Methods Biomed. Eng., 27(12):2035-2057, 2011.

[19] M.E. Moghadam, I.E. Vignon-Clementel, R. Figliola, and A.L. Marsden. A modular numerical method for implicit $0 \mathrm{~d} / 3 \mathrm{~d}$ coupling in cardiovascular finite element simulations. Journal of Computational Physics, 2012.

[20] P. Moireau, N. Xiao, M. Astorino, C. A. Figueroa, D. Chapelle, C. A. Taylor, and J-F. Gerbeau. External tissue support and fluid-structure simulation in blood flows. Biomech. Model. Mechanobiol., 11:1-18, 2012.

[21] A. Quarteroni, S. Ragni, and A. Veneziani. Coupling between lumped and distributed models for blood flow problems. Computing and Visualization in Science, 4:111-124, 2001.

[22] Y. Shi, P. Lawford, and R. Hose. Review of zero-d and 1-d models of blood flow in the cardiovascular system. Biomed Eng Online, 10:33, 2011.

[23] R. Temam. Sur l'approximation de la solution des equations de Navier-Stokes par la méthode des pas fractionaires I. Arch. Rational Mech. Anal., 32:135-153, 1969.

[24] I. E. Vignon-Clementel, C. A. Figueroa, K. E. Jansen, and C. A. Taylor. Outflow boundary conditions for 3d simulations of non-periodic blood flow and pressure fields in deformable arteries. Comp. Meth. Biomech. Biomed. Engrg., 13:625-640, 2010. 


\section{Contents}

1 Introduction 3

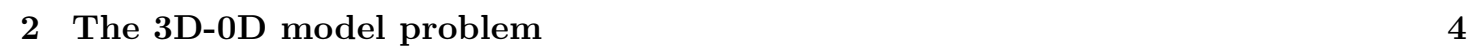

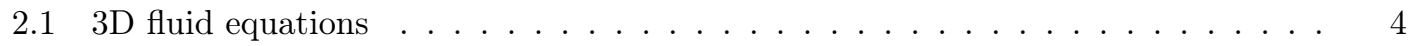

2.2 OD Windkessel model . . . . . . . . . . . . . . . . . . . . . . . . . . . 5

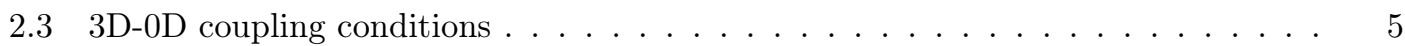

2.4 Time semi-discretization . . . . . . . . . . . . . . . . . . . . 6

$2.4 .1 \quad$ Fractional-step fluid time-marching . . . . . . . . . . . . . . . . . . 6

2.4 .2 Backward-Euler Windkessel time-marching . . . . . . . . . . . . . . . . . 7

2.5 Spatial discretization . . . . . . . . . . . . . . . . . 7

\begin{tabular}{|lll|}
\hline 3 & Fractional-step time-marching and 3D-0D coupling schemes & 7
\end{tabular}

3.1 Explicit 3D-0D coupling scheme . . . . . . . . . . . . . . . 7

3.1.1 Stability analysis $\ldots \ldots \ldots \ldots \ldots$

3.2 Implicit 3D-0D coupling scheme . . . . . . . . . . . . . . . . . . . . . . . . . . . . 10

3.2.1 Stability analysis $\ldots \ldots \ldots \ldots \ldots \ldots$. . . . . . . . . . . . . . . . . . . . . . . . . . . . . . 11

3.2 .2 Implementation details . . . . . . . . . . . . . . . . . . . . . . 12

3.2.3 Extension to higher order time-splitting schemes . . . . . . . . . . . . . . 13

3.2.4 Extension to more complex lumped parameter models . . . . . . . . . . . 14

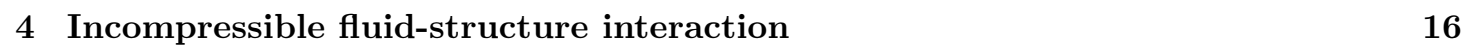

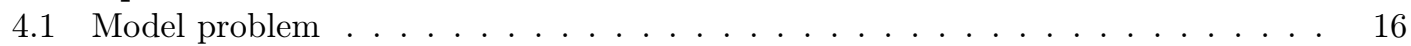

4.1 .1 Spatial discretization . . . . . . . . . . . . . . . . . 17

$4.1 .2 \quad$ Semi-implicit FSI scheme with implicit 3D-0D coupling . . . . . . . . . . 18

4.1.3 $\quad$ Semi-implicit FSI scheme with explicit 3D-0D coupling. . . . . . . . . . . 21

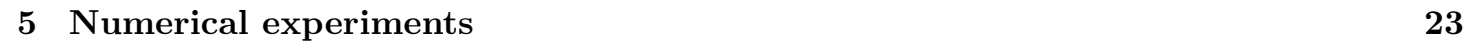

5.1 Blood flow in a patient-specific aorta . . . . . . . . . . . . . . . . . 23

5.2 Fluid-structure interaction in an idealized AAA . . . . . . . . . . . . . . . . . . . 25

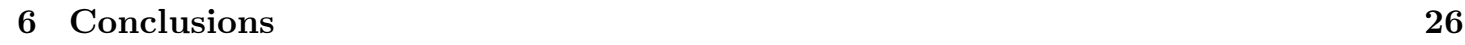




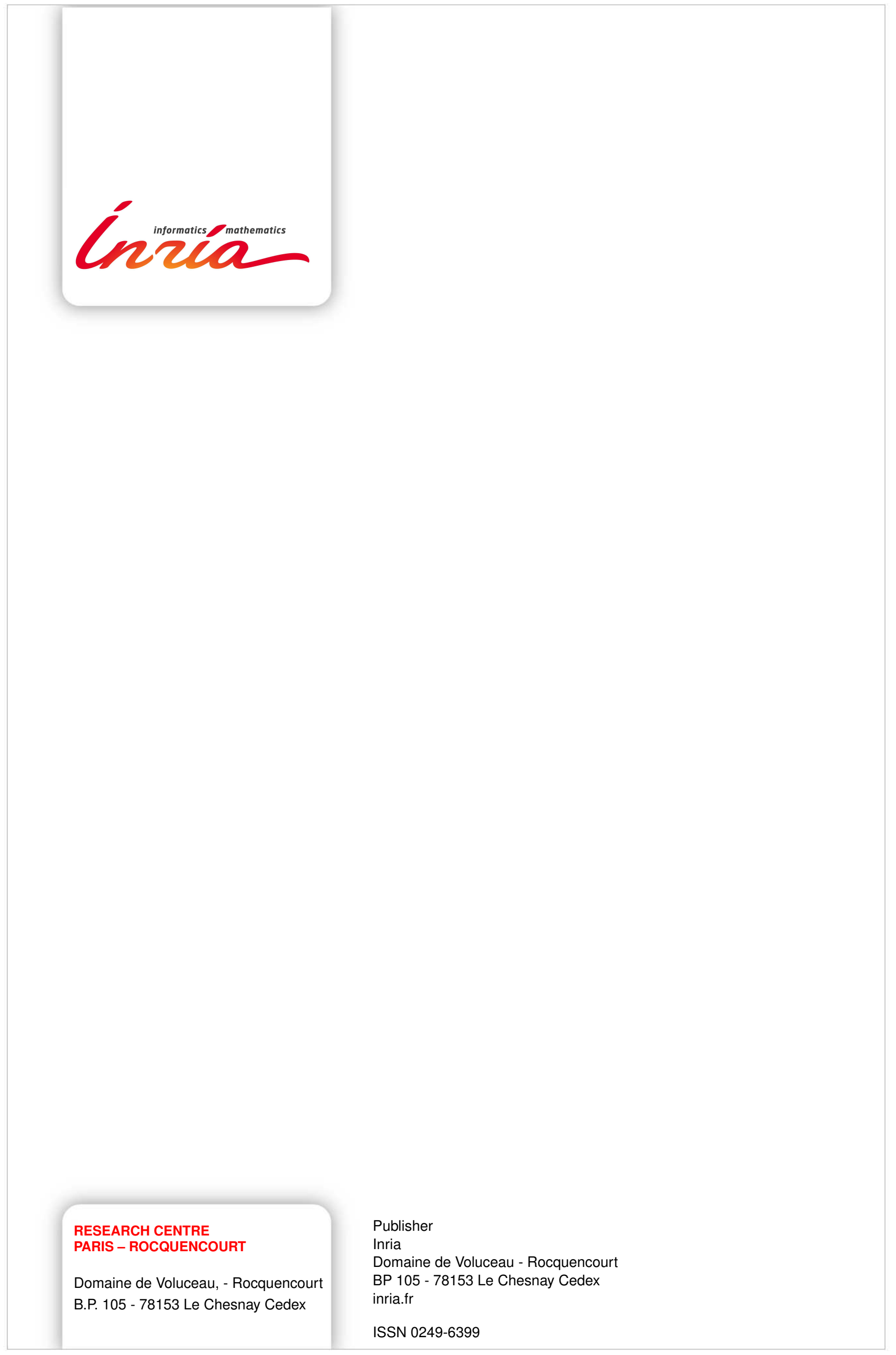

